\title{
The Science of Exoplanets and Their Systems
}

\author{
Helmut Lammer, Michel Blanc, ${ }^{2}$ Willy Benz, ${ }^{3}$ Malcolm Fridlund, ${ }^{4}$ Vincent Coudé du Foresto,,6 \\ Manuel Güdel, ${ }^{7}$ Heike Rauer, ${ }^{8}$ Stephane Udry, ${ }^{9}$ Roger-Maurice Bonnet, ${ }^{6}$ Maurizio Falanga, ${ }^{6}$ \\ David Charbonneau, ${ }^{10}$ Ravit Helled, ${ }^{11}$ Willy Kley, ${ }^{12}$ Jeffrey Linsky, ${ }^{13}$ Linda T. Elkins-Tanton, ${ }^{14}$ Yann Alibert, ${ }^{3}$ \\ Eric Chassefière, ${ }^{15}$ Therese Encrenaz, ${ }^{5}$ Artie P. Hatzes, ${ }^{16}$ Douglas Lin, ${ }^{17}$ Rene Liseau, ${ }^{18}$ \\ Winfried Lorenzen, ${ }^{19}$ and Sean N. Raymond ${ }^{20}$
}

\begin{abstract}
A scientific forum on "The Future Science of Exoplanets and Their Systems," sponsored by Europlanet* and the International Space Science Institute (ISSI) ${ }^{\dagger}$ and co-organized by the Center for Space and Habitability $(\mathrm{CSH})^{*}$ of the University of Bern, was held during December 5 and 6, 2012, in Bern, Switzerland. It gathered 24 well-known specialists in exoplanetary, Solar System, and stellar science to discuss the future of the fastexpanding field of exoplanetary research, which now has nearly 1000 objects to analyze and compare and will develop even more quickly over the coming years. The forum discussions included a review of current observational knowledge, efforts for exoplanetary atmosphere characterization and their formation, water formation, atmospheric evolution, habitability aspects, and our understanding of how exoplanets interact with their stellar and galactic environment throughout their history. Several important and timely research areas of focus for further research efforts in the field were identified by the forum participants. These scientific topics are related to the origin and formation of water and its delivery to planetary bodies and the role of the disk in relation to planet formation, including constraints from observations as well as star-planet interaction processes and their consequences for atmosphere-magnetosphere environments, evolution, and habitability. The relevance of these research areas is outlined in this report, and possible themes for future ISSI workshops are identified that may be proposed by the international research community over the coming 2-3 years. Key Words: Exoplanets-Disks-Planet formation-Stellar activity-Water originWater delivery-Habitability. Astrobiology 13, 793-813.
\end{abstract}

\footnotetext{
*http://www.europlanet-ri.eu.

†http://www.issibern.ch.

thttp://www.csh.unibe.ch/content/index_eng.html.
}

\footnotetext{
${ }^{1}$ Space Research Institute, Austrian Academy of Sciences, Graz, Austria.

${ }^{2}$ IRAP, Observatoire Midi-Pyrénées, UPS-CNRS, Toulouse, France.

${ }^{3}$ Physics Institute, University of Bern, Bern, Switzerland.

${ }^{4}$ ESTEC/ESA, Noordwijk, the Netherlands.

${ }^{5}$ LESIA - Observatoire de Paris, Meudon, France.

${ }^{6}$ International Space Science Institute (ISSI), Bern, Switzerland.

${ }^{7}$ Institute for Astronomy, University of Vienna, Vienna, Austria.

${ }^{8}$ Institut für Planetenforschung, Extrasolare Planeten und Atmosphären, DLR, Berlin, Germany.

${ }^{9}$ Observatoire de Genève, Geneva, Switzerland.

${ }^{10}$ Department of Astronomy, Harvard University, Cambridge, Massachusetts, USA.

${ }^{11}$ Department of Geophysics and Planetary Sciences, Tel-Aviv University, Tel-Aviv, Israel.

${ }^{12}$ Institute for Astronomy and Astrophysics, University of Tübingen, Tübingen, Germany.

${ }^{13}$ JILA, University of Colorado and NIST, Boulder, Colorado, USA.

${ }^{14}$ Department of Terrestrial Magnetism, Carnegie Institution for Science, Washington, DC, USA.

${ }^{15}$ Université Paris-Sud, Laboratoire IDES, CNRS, Orsay, France.

${ }^{16}$ Thüringer Landessternwarte Tautenburg, Tautenburg, Germany.

${ }^{17}$ University of California Observatories, Lick Observatory, Santa Cruz, California, USA.

${ }^{18}$ Earth and Space Sciences, Chalmers University of Technology, Gothenburg, Sweden.

${ }^{19}$ Institute of Physics, University of Rostock, Rostock, Germany.

${ }^{20}$ Laboratoire d'Astrophysique de Bordeaux, Bordeaux, France.
} 


\section{Introduction}

$T^{1}$ he International Space Science Institute (ISSI) in Bern, Switzerland, is an Institute of Advanced Study where international scientists can meet in a multi- and interdisciplinary setting to establish new scientific visions related to a widespread spectrum of disciplines from the physics of the Solar System and planetary sciences to astrophysics and cosmology and from Earth sciences to astrobiology. The main function of ISSI is to contribute to the achievement of a deeper understanding of results from different space missions, ground-based observations, and laboratory experiments, and to expand upon those results through multidisciplinary research in the framework of international teams, workshops, working groups, and forums.

International Space Science Institute forums are informal and free debates among some 15 to 25 high-level participants on open questions of scientific nature or science policy matters. Forums do not necessarily lead to formal recommendations or decisions. They are generally held a few times each year at ISSI for 2 days. During December 5 and 6, a 2day scientific forum that focused on "The Future Science of Exoplanets and Their Systems," sponsored by ISSI through the EU FP7 Research Infrastructure project Europlanet, was organized and brought together 24 globally renowned scientists who have contributed to exoplanetary, Solar System, and stellar science over the course of the past decades to discuss the future of the fast-expanding field of exoplanetary research. Now, more than 860 exoplanets are known, and in the near future observing facilities will become extremely powerful. After the pioneering discovery of the first transiting super-Earth CoRoT-7b by the CoRoT space observatory in 2009 (Léger et al., 2009), more exoplanets of that category have been discovered by ground-based projects and NASA's Kepler satellite (Borucki et al., 2011). The first potentially habitable planets have already been discovered around $\mathrm{M}$ dwarf stars (e.g., Selsis et al., 2007; von Bloh et al., 2007) and recently around a K star (Borucki et al., 2013). More potentially habitable exoplanets will be discovered in the near future. Because such discoveries will have significant impact on modern planetary science and society as a whole, this particular ISSI forum's main goal was to debate and discuss how observed and modeled planetary properties can be represented in the most useful and efficient way. By doing this, questions such as those listed below may be solved in the near future:

- Is there a typical architecture of planets and/or planetary systems?

- What is driving planet formation and evolution?

- What is the relevance of planet formation and a system's impact history to the evolution of Earth-like habitats and planets in general (i.e., its outgassing history, hence relationships between interiors and atmospheres, climate stabilization for habitable planets related to different rock/ice/atmosphere ratios, etc.)?

By dealing within a multi-parameter space, the most relevant observables that can be described with a minimum of complexity to understand planetary evolution were debated and identified. The discussions addressed the following key objectives:

- The first objective was related to discussions of exoplanetary properties, which focused on observables and minimum complexity models in theory (i.e., planet formation, dynamic, interior, atmospheres, etc.), as well as on interdisciplinary aspects.

- The second objective focused on exoplanetary environments with emphasis on disk evolution, stellar radiation, and plasma properties.

- The third objective involved detailed discussions on the origin and delivery of the earliest atmospheres and planetary water inventories.

- The fourth and final objective focused on the necessary requirements for observationally based data, as well as lab data, theoretical models, and ground- and spacebased proposed and selected projects/missions.

Along with these objectives, related discussions led to the identification of important research areas in which only an interdisciplinary approach, as addressed in specialist workshops and related activities, would enhance knowledge in the field of exoplanetary research. This report on the science of exoplanets and their systems summarizes the main results of this particular ISSI forum.

\section{Exoplanetary Properties}

The fast-growing field of exoplanetary science is currently in an observationally driven phase in which theoretical models, such as photochemical, thermospheric, and hydrodynamic atmospheric codes, Monte Carlo and test-particle tools, and various numerical transit algorithms used to characterize newly discovered planets are of use only when interpreting these observations. Due to the complex nature of planetary systems, it is highly unlikely that a single theory can explain a newly discovered system. Therefore, several models must be developed so that the important and most relevant mechanisms and their outcomes can be understood within the parameter space given by the observations.

\subsection{Detection methods}

The main methods that have been used so far in the discovery of exoplanets are as follows:

- the Doppler method,

- the transit method,

- direct imaging,

- gravitational microlensing,

- astrometry.

Most exoplanets have been discovered by the Doppler method, which monitors the change in Doppler shift of the spectral absorption lines of the exoplanet's host star. From the Doppler shift, the minimum mass of the observed target planet can be determined, as well as the body's semimajor axis and orbital eccentricity. Radial velocity measurements on stars related to the search and confirmation of exoplanets are at present mainly performed with the $3.6 \mathrm{~m}$ ESO High Accuracy Radial Velocity Planet Searcher (HARPS); the Very Large Telescope spectrographs UVES, FLAMES, and CORALIE; the $1.93 \mathrm{~m}$ SOPHIE spectrograph in the Observatoire de Haute Provence; the Coudé echelle spectrograph from the $2 \mathrm{~m}$ telescope in Tautenburg (TLS), Germany; and spectrographs at the McDonald and Keck observatories. The current limiting precision reached by the HARPS project is $\sim 0.5 \mathrm{~m} \mathrm{~s}^{-1}$, while the signal for super-Earths to Earth-mass planets orbiting a Sun-like star at 1 AU would be $\sim 0.1 \mathrm{~m} \mathrm{~s}^{-1}$ 
to $0.06 \mathrm{~ms}^{-1}$. However, it should be noted that the first Earth-mass exoplanet within a close-in orbit of $\alpha$ Centauri B has been discovered recently (Dumusque et al., 2012). The amplitude of this signal is $0.5 \mathrm{~m} \mathrm{~s}^{-1}$, which is significantly below that of many other astrophysical signals, including binaries, stellar rotation, and long-term magnetic activity.

The second powerful method in exoplanet detection is the transit method, which monitors the change in brightness of the planet's host star. With this method, the radius of an exoplanet and its semimajor axis can be determined. It should be noted that not all exoplanets discovered by the Doppler method are in an orbit around their stars such that they can also be seen by transit observations (e.g., $<10 \%$ transit probability for close-in planets). But exoplanets that are discovered by transits can be re-observed by the Doppler method; thus their mass can also be determined if the precision is above the current signal threshold. The discovery of CoRoT-7b, the first small rocky exoplanet with measured radius and mass (Léger et al., 2009) and therefore with a known density, has opened up a new research era. While the European Convection, Rotation and planetary Transits (CoRoT) space observatory has demonstrated that exoplanets can be observed via transits from space, the CoRoT satellite, due to its mission design, cannot discover Earthsized exoplanets within the habitable zones of their host stars. This important task has been taken over by NASA's Kepler satellite, which has been in space since March 2009 (e.g., Borucki et al., 2011). The current limiting precision for the transit method is given by Kepler with $\sim 20 \mathrm{ppm}$. The Earth-Sun transit signal is $\sim 80 \mathrm{ppm}$. Compared to the Doppler method, which cannot measure Earth-mass bodies at $1 \mathrm{AU}$ at present, Earth-sized exoplanets at a distance of about 1 AU can be discovered by the Kepler space observatory. From the discoveries of exoplanetary systems such as Kepler-20, it is known that Earth-sized planets are interspersed among larger Neptune-sized bodies. The Kepler mission has discovered about 2300 exoplanet candidates so far, most of them smaller than Neptune, that could potentially be used for the determination of the occurrence rate of Earth analogues. It should also be noted that the hunt for exoplanets below the jovian class and neptunian class by Kepler is supported by ground-based transit projects such as the MEarth Project in the USA. The MEarth Project is a photometric ground-based survey that monitors about 2000 nearest $M$ dwarfs for transiting super-Earths with orbits inside close-in habitable zones. The most interesting discovery of this project so far is a sub-Neptune/super-Earth-class exoplanet with the size of $\sim 2.7$ Earth radii with an orbital period of 1.6 days around the small, faint star GJ 1214 (Charbonneau et al., 2009).

Direct imaging is the third method for the detection of exoplanets by the determination of the band-integrated luminosity and the projected planet-star separation. From the few directly detected exoplanetary systems, it can be ex- pected that these systems are very young and widely separated. This method does not permit a direct measurement of the mass or size of the imaged planets.

The fourth method in the detection of exoplanets is gravitational lensing, which monitors the change in brightness of a distant star because its light is bent and focused by gravity when a star with an orbiting exoplanet passes between the distant star and Earth. From this method, the mass of the exoplanet and its semimajor axis can be determined. So far 14 exoplanets have been discovered with the gravitational microlensing method. The disadvantage of this method is that, in general, follow-up observations are not possible. It should be noted that microlensing is the only practical method that allows for discovery of the population of gas and ice giants significantly beyond the ice line (see Fig. 6 in Gaudi, 2012). The science programs of ESA's Euclid and NASA's planned WFIRST fundamental physics space missions contain microlensing searches of exoplanets within their science cases.

Astrometry can be seen as the fifth method for exoplanet detection. This method consists of precisely measuring a star's location in the sky and its related position changes over time. In the near future, ESA's Gaia all-sky survey astrometry space mission will also contribute to the statistics of exoplanets via astrometry (Sozzetti, 2010). For example, the Gaia data, over the next decade, will allow us to significantly refine the understanding of the statistical properties of extrasolar gas giants and will enhance our knowledge of potential exoplanet host star parameters. Also, asteroseismic studies will yield the age of exoplanet host stars.

\subsection{Observables and science}

With this brief survey of the present-day exoplanet detection methods, it should be understood that the Doppler and transit methods will dominate exoplanet detection in the near future in comparison to detection by direct imaging, gravitational microlensing, and space astrometry. Table 1 compares the current limiting precision of the Doppler and transit methods and the corresponding necessary values for an EarthSun signal at 1 AU orbit location. Therefore, a short summary of the science that can be obtained by the combination of the transit and Doppler methods is addressed below.

Exoplanet mean density. By knowing the radius and mass of an exoplanet, the mean density can be calculated. The present data base already shows that planet densities span a wide range for any given planet mass. This indicates the existence of very different types of planets with similar masses. A big surprise from the available observations of this kind is that there are many close-in hot Jupiters that have inflated radii compared to their expected radii from radiusmass models that are used for Solar System gas giants. Furthermore, a detailed investigation of the first discovered super-Earths with known size and mass indicates that many

Table 1. Current Possible Precision Limits and Necessary Signals for the Detection of a "Super-Earth" and an Earth-Analog Planet at an Orbit at 1 Au around a Sun-like Star

\begin{tabular}{lcc}
\hline Method & Limited precision & "Super-Earth" - Earth-Sun signal at 1 AU \\
\hline Doppler method (mass) & $0.5 \mathrm{~m} \mathrm{~s}^{-1}$ & $0.1-0.06 \mathrm{~m} \mathrm{~s}^{-1}$ \\
Transit method (radius) & $20 \mathrm{pm}$ & $80 \mathrm{ppm}$ \\
\hline
\end{tabular}




\section{a}

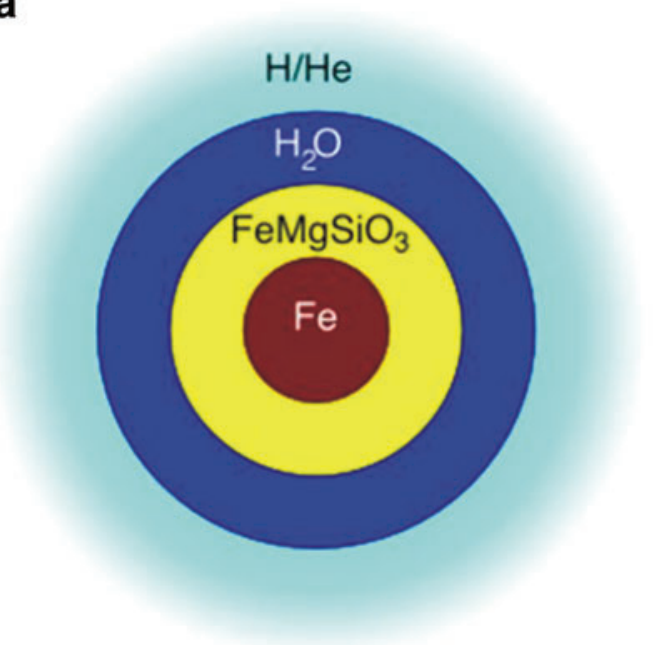

b

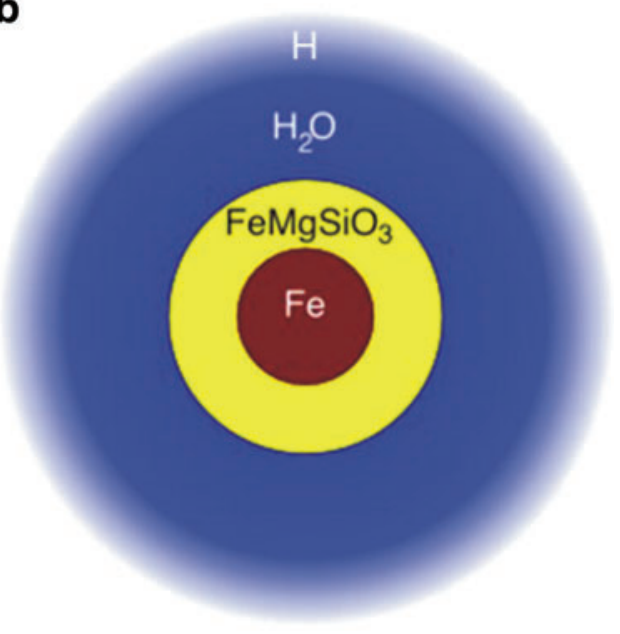

FIG. 1. Illustration of two possible scenarios for the structure of the low-density super-Earth GJ 1214, which can be derived from the radius-mass relation within the present uncertainties. Left scenario (a) would correspond to a miniNeptune with a dense core that is surrounded by a primordial or outgassed dense hydrogen envelope. Right scenario (b) corresponds to a low-density core that is surrounded by a huge amount of $\mathrm{H}_{2} \mathrm{O}$ and a thin hydrogen envelope (courtesy of D. Charbonneau). Color images available online at www.liebertonline.com/ast

of them are most likely surrounded by hydrogen envelopes or dense hydrogen-rich volatiles that comprise a few percent of their masses (e.g., Lissauer et al., 2011; Ikoma and Hori, 2012; Lammer et al., 2013). The existence of such envelopes indicates that these planets' nebula-based or outgassed protoatmospheres were not dispelled (Kuchner, 2003; Lammer, 2013; Lammer et al., 2013). These discoveries are very important and are closely linked to the formation of the whole system; the host stars' early radiation; and the plasma environment that is linked to the nebula lifetime, the inner structure, related physics of the exoplanets, and, with regard to super-Earths and Earth-sized exoplanets, habitability. Although the available data suggest that more massive and slightly larger planets compared to Earth with orbital distances >0.02 AU (around G stars) are more likely miniNeptunes than pure rocky planets with tiny Earth-like atmospheres, the present error bars in the radius-mass relation are too large and must be reduced. As can be seen in Fig. 1, a good example is the low-density super-Earth GJ $1214 \mathrm{~b}$ whose radius-mass related structure can be fitted by a dense core model that is surrounded by a dense primordial based $\mathrm{H} / \mathrm{He}$ envelope or outgassed $\mathrm{H}_{2}$ as well as by a lowdensity core surrounded by a deep $\mathrm{H}_{2} \mathrm{O}$ ocean and a thin hydrogen envelope at the top (Adams et al., 2008; Rogers and Seager, 2010; Nettelmann et al., 2011; Berta et al., 2012).

Exoplanetary atmospheres. Planetary transits offer the opportunity not only to discern the size of an exoplanet but to study its atmosphere as well, which to date has not been possible for non-transiting planets. As illustrated in Fig. 2, the radiation from the exoplanet host star is transmitted through its atmosphere during the transit, while the thermal radiation and the reflected light from the planet disappear and reappear during the secondary eclipse. By measuring the planet-to-star flux ratio as a function of wavelength, spectra of the planetary atmosphere are obtained (Charbonneau et al., 2008; Grillmair et al., 2008).
Vidal-Madjar et al. (2003) observed the transiting exoplanet HD 209458b with the Hubble Space Telescope (HST) Space Telescope Imaging Spectrograph (STIS) and discovered an intensity drop in the stellar Lyman- $\alpha$ line, which was surprisingly large, considering that the atmosphere of a planet occulted only $1.5 \%$ of the star. Subsequent observations at low spectral resolution with the HST STIS/ Advanced Camera for Surveys (ACS) have also confirmed that the transit depth in Lyman- $\alpha$ is significantly greater than the transit depth due to the planetary disk alone (Ben-Jaffel, 2007; Ben-Jaffel and Sona Hosseini, 2010). Recently, another observation of an extended upper atmosphere due to Lyman- $\alpha$ absorption during the transits of a second short periodic Jupiter-type gas giant, HD 189733b, was reported (Lecavelier des Etangs et al., 2010, 2012). From these observations, it can be concluded that hydrogen atmospheres of hot Jupiters expand up to their Roche lobes and that close-in

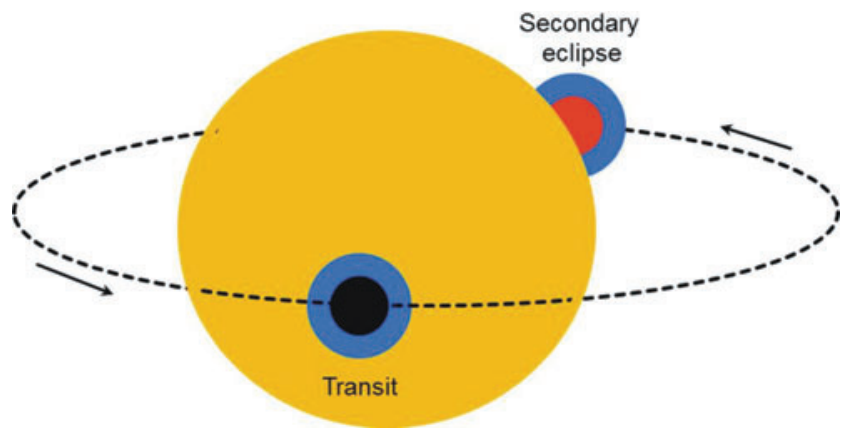

FIG. 2. Illustration of exoplanetary atmosphere effects during the transit and secondary eclipse of an exoplanet. During the transit, the radiation from the star is transmitted through the planet's atmosphere, while during the secondary eclipse the thermal radiation and reflected light from the planet disappear and reappear. Color images available online at www.liebertonline.com/ast 
gas giants are surrounded by cometary-type hydrogen clouds as predicted by Schneider et al. (1998). Applied hydrodynamic models by Yelle (2004), García Muñoz (2007), Penz et al. (2008), and Koskinen et al. (2013a, 2013b) support this hypothesis as well as independent HST/STIS and Cosmic Origins Spectrograph (COS) observations where carbon, oxygen, and silicon were observed beyond the Roche lobe of HD 209458b (Vidal-Madjar et al., 2004; Linsky et al., 2010) and various metals around the Roche lobe of WASP-12b (Fossati et al., 2010).

The detection and investigation of extended upper atmospheres around exoplanets of all types provide very promising insights into the interaction of the host star's plasma environment with the planet itself. Lammer et al. (2011) and Lammer (2012) showed that it should be possible to detect extended upper atmospheres and their related hydrogen coronae even around small, Earth-sized exoplanets orbiting cool M-type stars with space observatories such as the World Space Observatory-UV (WSO-UV) (Shustov et al., 2009), which are currently under development.

From transit spectroscopy by follow-up observations with the Spitzer Space Telescope, even molecules (i.e., $\mathrm{H}_{2} \mathrm{O}, \mathrm{CH}_{4}$, etc.) in hot Jupiter atmospheres have been inferred (e.g., Tinetti et al., 2007; Swain et al., 2009; Beaulieu et al., 2010). By measuring the combined star-planet flux for determining a longitudinally resolved emission map as a function of wavelength, the day-night circulation of an exoplanetary atmosphere can be discerned (Knutson et al., 2007, 2009). Transmission spectra modeling on the above-discussed transiting super-Earth GJ 1214b can also be used for clarifying the uncertainties in the planet's structure. Model simulations of the GJ 1214b WFC4 transmission spectrum by
Miller-Ricci and Fortney (2010) ruled out the dense core primordial hydrogen envelope scenario of Fig. 3a at $8.3 \sigma$, while their result agrees well with $\mathrm{H}_{2} \mathrm{O}$ fractions $>20 \%$ as illustrated in Fig. 3b. This example shows that the structure of this exoplanet can be characterized to a certain point if the radius, the mass, and the transmission spectrum are known. Although the observations contain uncertainties, the results clearly suggest that this particular planet may be a water world that is surrounded by a $\mathrm{H}_{2} \mathrm{O}$ atmosphere and a hydrogen corona. Unfortunately, due to the brightness properties of the host stars of the known exoplanets, only a tiny fraction, which includes GJ 1214b, of transmission spectra can be observed. Perhaps the James Webb Space Telescope (JWST) and the ground-based ESO Extreme Large Telescope (ELT), the Giant Magellan Telescope, and the Thirty Meter Telescope projects can observe atmospheric species for cooler super-Earths in a similar way as the HST, Spitzer, and ground-based projects have for some hot Jupiters and Neptunes.

Spin-orbit misalignment determination. By investigating a transiting planet with a known mass, the planet's spin-orbit alignment can be determined by the Rossiter-McLaughlin effect (Gaudi and Winn, 2007; Albrecht et al., 2012). From such studies, it is known that many hot Jupiter systems are misaligned, which has widened the picture of planetary migration models.

Planet-radius distribution. From the survey of the discovered transiting exoplanets and Kepler's discovery of $\sim 2300$ planet candidates, a statistic related to the planet-radius distribution can be determined. From these discoveries, we

FIG. 3. Illustration of the recently selected TESS (top left) and planned PLATO (top right) future transit search missions for planets in orbits around nearby bright host stars. Below, illustration of the Swiss-led CHaracterizing ExOPlanets Satellite (CHEOPS) recently selected by ESA within their small mission program. CHEOPS main science aims will be the determination of the radius-mass relation of exoplanets, the identification of significant gaseous envelopes around the planet's core, and the probing of known hot Jupiter atmospheres with the intent to study the physical mechanisms and efficiency for energy transport from the dayside to the nightside of the planet (NASA/ESA). Color images available online at www.liebertonline .com/ast

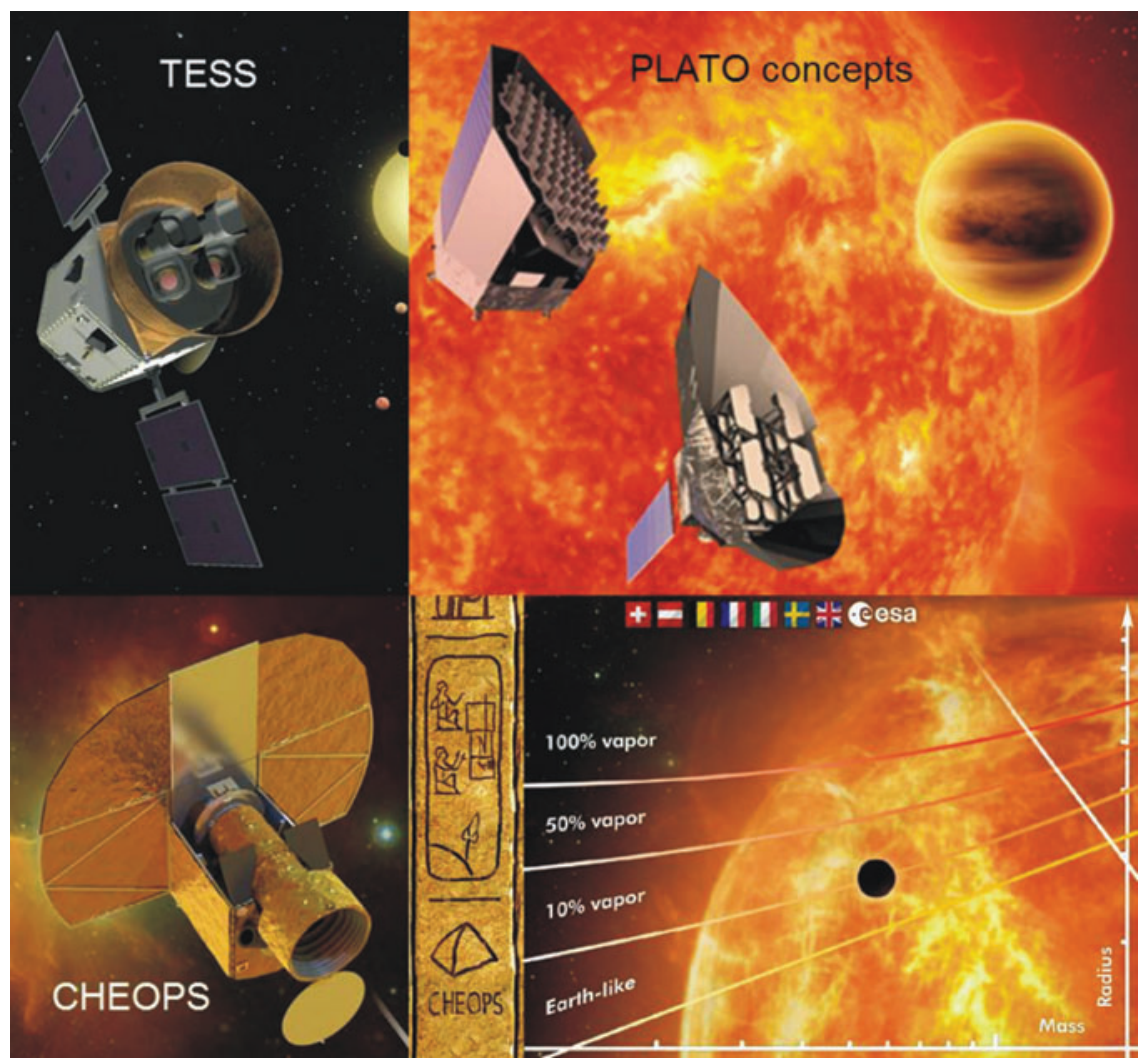


find the power law $\mathrm{dN} / \mathrm{d} \log R_{\mathrm{pl}}=k R_{\mathrm{pl}}{ }^{\alpha}$ (Batalha et al. 2012; Howard et al., 2012) with coefficients $k=3.8 \pm 0.3$ and $\alpha=-2.01 \pm 0.09$, which indicates that exoplanets with small radii are much more numerous compared to Jupiter-like gas giants. From the available Kepler survey, an occurrence rate can be derived of planets between $0.4-4 R_{\oplus}$ within orbital periods $<50$ days of 0.91 planets per cool star. The occurrence rate of Earth-sized planets in the habitable zone of cool stars is $\sim 0.1$ planets per cool star. From this statistic, we find with $\sim 95 \%$ confidence that there is a transiting Earth-sized planet in the habitable zone of a cool star within 23 pc (Dressing and Charbonneau, 2013).

\subsection{Improvements for the future and open questions to be solved}

Although there are more than 850 exoplanets known, and we know the size and mass of about 290, the atmosphere of these planets can only be studied for a smaller fraction of the total number due to the faintness of their host stars (e.g., Seager and Deming, 2010). With the ever-increasing number of detected transiting planets, our knowledge of the physical parameters for characterizing planets has increased significantly, which shows that planets are much more diverse than we expected from what we know of the Solar System. This increasing understanding of planet populations has been triggered by transiting planets for which we can directly measure their bulk parameters (radius, mass, mean density) with the Rossiter-McLaughlin effect to determine orbit alignment, measure effective temperatures and albedos, and finally even detect atmospheric molecular absorptions. Exoplanetary atmosphere observations are evolving as a separate research field that is now firmly established, with more than two dozen atmospheres of hot Jupiters observed. Highlights include

- the detection of molecular spectral features,

- the observation of day-night temperature gradients,

and

- the constraints on vertical atmospheric structure.

The success of ground-based surveys and, in particular, the CoRoT and Kepler space missions has also shown that transits are an efficient method to detect large numbers of planets with well-known parameters. It is true that efficient transit detections are biased to short orbital periods due to the low geometrical transit probability. This disadvantage over the radial velocity method is, however, not true when one considers searches for small terrestrial planets at intermediate to large orbital distances for which also radial velocity detections require a huge investment of observing time per planet. When it becomes possible to determine the entire parameter range for each detected exoplanet, including terrestrial planets, we will enter a new era of planetary science. To date, this is possible only for planets orbiting the brightest host stars among the almost thousand detected exoplanets. To date, the results of the CoRoT and Kepler space observatories as well as the ground-based surveys have mainly contributed to exoplanet statistics, but their discoveries are not ideal for spectroscopic follow-up observations because the targets are too distant. The overlap between planets that have been discovered by the Doppler method and transiting planets of bright stars is very small. There are many radial velocity planets with known mass around brighter nearby stars, while transiting exoplanets so far are discovered mainly around fainter stars. This fact results in a target problem for future exoplanetary atmosphere characterization projects.

All the participants of the ISSI forum agreed that more detections of exoplanets around bright and nearby stars are necessary in order to characterize large numbers of exoplanetary atmospheres more accurately than at present. When investigating habitable planets that may have evolved to be comparable to Earth, such detection surveys must include intermediate orbital distances, where we expect temperate planets. Two space missions are currently under study for the detection of exoplanets around nearby bright stars: the NASA Transiting Exoplanet Survey Satellite (TESS) mission in the USA and ESA's PLAnetary Transits and Oscillations of stars (PLATO) mission in Europe.

The TESS space observatory has been selected recently by NASA for an all-sky survey of bright, nearby stars; the mission should launch in 2017. Its prime target range is transiting Earths and super-Earths. TESS will survey bright host stars at 4.5-12 mag. It will survey F, G, and $\mathrm{K}$ stars and will also scan bright $M$ stars within 50 pc. In total, about 500,000 stars will be observed during 2 years' mission lifetime. Altogether, it is expected that TESS will discover more than 1000 new exoplanets, and several hundreds of them will be Neptune-sized or smaller. TESS will operate in a step-andstare mode to cover the whole sky. The observational coverage of each field (hence of the orbital planet periods scanned for) will depend on its position in the sky. Most of the detections will be made for short-period planets, but for M dwarf stars planet detections up to their habitable zones will also be possible. Such bright planetary systems will also be prime targets for, for example, spectroscopic follow-up characterization of their atmospheres by the JWST and planned future projects such as ESA's Exoplanet Characterisation Observatory (EChO).

The JWST will also probe exoplanetary atmospheres as Spitzer did (e.g., Seager et al., 2009) but will most likely focus on potential habitable planets around bright $\mathrm{M}$ stars. Favorable transiting planetary systems will most likely be discovered by TESS and PLATO. However, the atmosphere observations will require tens to hundreds of hours of JWST time per planet. Long follow-up observation time may be a problem because the JWST is not an exoplanet mission per se.

The next-generation planet finder mission PLATO has been proposed to ESA for the M3 mission launch opportunity in 2022/24. It is planned as a large-scale detection survey that will provide statistically relevant numbers of well-characterized exoplanets with highly accurate bulk parameters and ages, in particular in the Earth to super-Earth domain around bright host stars. PLATO would be the first mission to make systematic use of both the transit planet search technique and asteroseismology of the planet hosts. This will not only provide highly accurate parameters for planets and their host stars but also allow for determination of the age of planetary systems on a large scale. The PLATO prime target range is 4-11 mag. This bright target range allows surveying Sun-like F, G, and K stars as well as the brightest and nearest $M$ dwarfs for terrestrial planets. PLATO will have the capacity to detect thousands of Neptune-sized and smaller planets, and hundreds of these 
planets will be in the super-Earth regime, including planets in the habitable zone of bright Sun-like stars. The telescope combines a step-and-stare observing strategy with two longterm (up to 3 years) field observations. With a total mission lifetime of $\sim 6$ years, $\sim 50 \%$ of the sky will be scanned for new exoplanets. Its detection range therefore targets

- at intermediate orbital distances, up to the habitable zone of Sun-like stars, to significantly expand the number of well-characterized cool and potentially habitable exoplanets;

and

- large numbers of terrestrial planets in this intermediate orbital distance range with well-known bulk parameters and ages as the prime goal for a detection survey operating in the next decade.

Due to technological differences and hence observational capabilities between TESS and PLATO, the selection of these missions has an impact on the accuracy of the observations related to the target planet's orbit location. In the case of expected discoveries by TESS, the first accurate characterization of exoplanetary atmospheres by a future characterization project will focus on exoplanets that have their orbits inside $\mathrm{M}$ star habitable zones. In the case of PLATO, future characterization missions would probe planetary atmospheres in orbital distances within habitable zones around Sun-like stars.

Smaller numbers of presently known or soon-to-be discovered exoplanets around brighter stars will also be studied with ESA's recently selected first small mission, the CHaractizing ExOPlanets Satellite (CHEOPS). A Swiss-led, ESA-supported, European endeavor, CHEOPS is a small space telescope that is designed for the follow-up observation of exoplanets discovered within the overlap of transits and Doppler observations. Ground-based high-precision Doppler spectroscopic surveys have been carried out during recent years, and hundreds of stars with exoplanets in the super-Earth to Neptune mass range $\left(1<M_{\mathrm{pl}} / M_{\oplus}<20\right)$ have been identified. These surveys will continue into the foreseeable future. The characteristics of these stars (brightness, low activity levels, etc.) and the knowledge of the planet ephemerides make them ideal targets for ultrahigh-precision photometric measurements from space with CHEOPS. This mission, which will be launched in 2017, is the only space observatory with the capacity to follow up all these targets for precise radius measurements in the near future.

The new generation of ground-based transit surveys (e.g., NGTS) should be capable of reaching 1 mmag precision on $\mathrm{V}<13$ mag stars, so that new targets for CHEOPS can be expected. By the end of 2017, NGTS will provide of order 50 targets with a radius less than 6 Earth radii for which CHEOPS will be able to measure radii to a precision of $10 \%$. The studied planets can be selected for further investigations with the JWST, ELTs, and so on. The most interesting targets for CHEOPS will be hot Neptunes and super-Earths. From the discussions above, it is clear that the main challenge is that, if smaller planets are discovered, the related error bars become more and more relevant. With the space observatories briefly mentioned above and the currently developed instruments that will be installed on available or planned ground-based telescopes, the error bars in size and mass determinations will be minimized.
Launched and future Solar System missions, such as Cassini, Juno, or JUICE, will certainly contribute to our understanding of gas giants but will not deliver the whole answer. Cassini obtains gravity data of high accuracy around Saturn, which are currently studied and analyzed for a better understanding of the core-atmosphere structure of the planet.

Although we had a spacecraft near Jupiter, it is not known so far whether the closest gas giant has a core, mantle, and envelope (three-layer structure) or only a core and an envelope (two-layer structure) (Nettelmann, 2011). However, the uncertainties due to the unknowns in the applied equation of state are becoming less important due to advances in ab initio modeling techniques and computer power (e.g., French $e t$ al., 2012; Nettelmann et al., 2012). Juno will set tighter constraints on Jupiter's core mass and internal structure, including the planet's water abundance.

Therefore, the expected results from the Juno mission will contribute to our understanding of Jupiter's origin and, via the $\mathrm{H}_{2} \mathrm{O}$-aboundance, possibly the physical/chemical conditions in the nebula and the history of volatiles. The obtained data and their properties can then be related to giant planet-formation theories. From the exoplanet surveys, we know that Uranus- and Neptune-type planets seem to be a very common planetary category in the Galaxy. Future Solar System missions to one of these planets would indeed help to understand what exoplanets of this kind are made of.

There are still many open questions regarding Uranus and Neptune. Their internal structure and compositions are still poorly understood, and in addition they differ in other physical parameters such as the inferred atmospheric composition, obliquity, and thermal emission. It is therefore clear that further investigation of the origin, evolution, and internal structure of intermediate-mass planets is desirable. All these open questions are directly related to the real boundary between gasrich planets and so-called terrestrial planets (see Fig. 4), where life-forms may evolve on the planet's ocean/surface as they did 3.5-3.8 Gyr ago on Earth. A detailed observation and study of this boundary bears directly on fundamental open questions related to planet formation such as

- runaway gas accretion in the core accretion scenario or the loss of primordial $\mathrm{H}-\mathrm{He}$ atmospheres

and

- constraints on possible planet migration paths followed during formation and evolution for planets where the clear presence of a massive gaseous envelope cannot be discerned.

These are key habitability questions that have a direct link to a possible architecture of habitable planets and the evolution of Earth-like class I habitats (Lammer et al., 2009; Lammer, 2013), where life as we know it can evolve. To solve these questions, the theoretical efforts combined with the above-mentioned enhancements in observables related to the following points have to be addressed:

- combining planet-formation theories with the formation environments;

- understanding the formation of intermediate-mass planets;

- making more accurate measurements of planetary parameters for planets with larger orbital locations; 


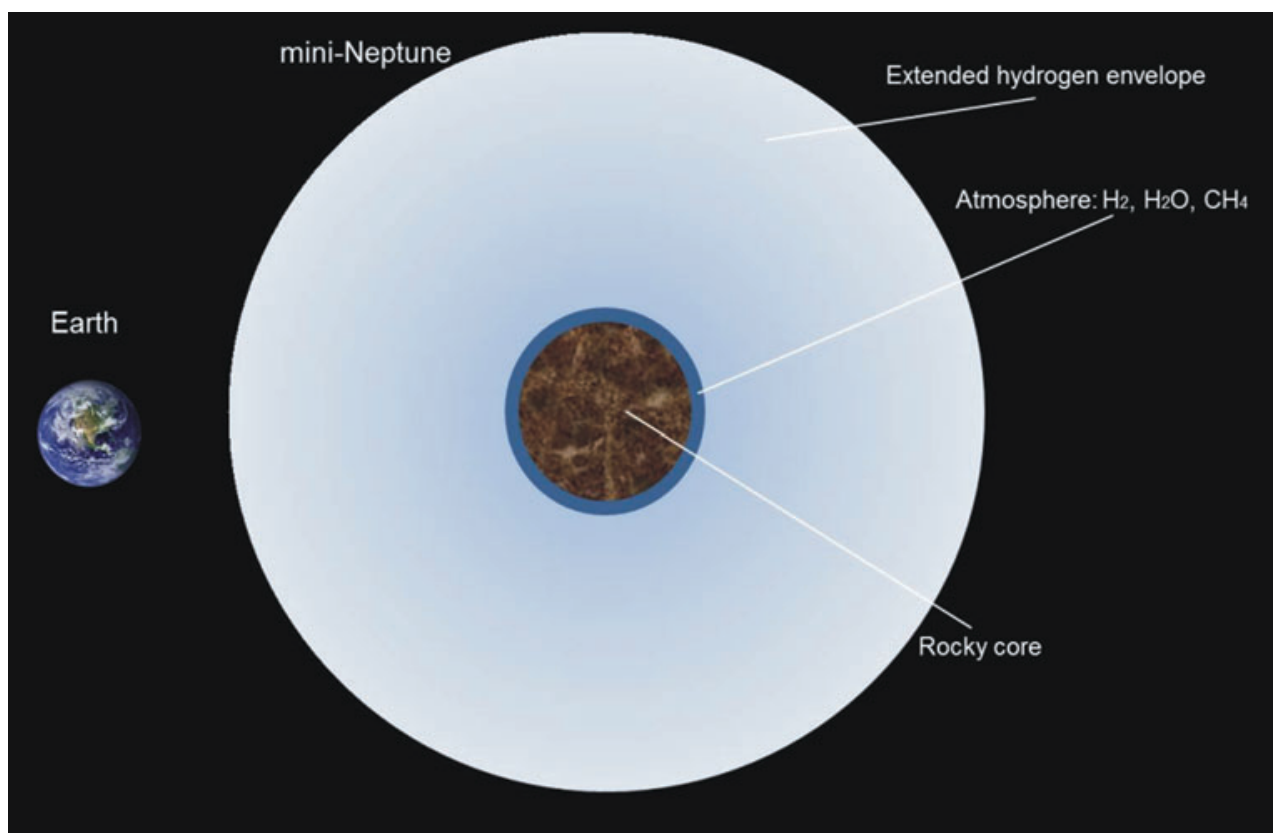

FIG. 4. Illustration of Earth and a super-Earth that mimics a mini-Neptune because it may not have lost its nebula-based captured hydrogen envelope or outgassed hydrogen-dominated volatile-rich protoatmosphere. Color images available online at www.liebertonline.com/ast

- combining exoplanet statistics, hence ensemble parameters, with predictions from planet-formation models; and

- obtaining the dimensionless Love number parameters that determine the rigidity of a planetary body and the susceptibility of its shape to change in response to a tidal potential, which would result in a better understanding of the planet's interior (Kramm et al., 2012).

For technological reasons, there is at present a bias in the characterization of exoplanets within close orbit locations around their host stars. These close-in planets, however, certainly do not represent the main populations, and additional complex processes such as heating and interaction processes with the host star will influence the evolution of such planets.

Therefore, it is important to obtain data from planets on orbital locations that are farther out. Theoreticians who model planetary composition and structure should investigate whether first-order uncertainties in their models can be separated out, for instance, to obtain information if a planet has a three-layer or two-layer structure. It is important to point out that it makes little sense to apply a very complex theoretical model if the input parameter space is so uncertain that every result can be hypothetically possible-including unrealistic ones. It is very important to understand how to quantify the theories within the available constraints and parameter space. For instance, to understand in an interdisciplinary way how planets such as those discovered in the densely packed Kepler-11 system originated and evolved would involve incredible theoretical efforts. Thus, the forum participants agreed that, with regard to theory, it is important to know to what extent first-order uncertainties in theoretical models can be solved with high-precision radius and mass measurements.

\section{Disk Evolution and Origin of Planets, Their Atmospheres, and Water Inventories}

Planet formation is closely connected to star formation and early stellar evolution (see e.g., Bodenheimer, 1997; Mannings et al., 2000; Wuchterl et al., 2000; Boss, 2003). Stars form from collapsing clouds of gas and dust. The collapse leads to the formation of the protostar in the center, which contains most of the mass of the cloud, and a circumstellar disk, which retains most of the angular momentum of the cloud. Because stars have various disk sizes and masses, there is no reason that a given star has given disk properties (Williams and Cieza, 2011). Thus, a huge variation can be expected. In the Solar System, the circumstellar disk is estimated to have had a mass of a few percent of the Sun's mass. The planets form from the material in the circumstellar disk, which is at this stage also referred to as the protoplanetary disk.

\subsection{Protoplanetary disk evolution}

Most protoplanets are formed within the accretion disk during the nebula lifetime, which lasts between $\sim 10^{6}$ and $10^{7}$ years (e.g., Montmerle et al., 2006). After $10 \mathrm{Myr}$, most nebular gas is evaporated from the system. Because the accretion disk contains about $99 \%$ gas and $1 \%$ dust, planets can accumulate nebular gas (e.g., Ikoma and Genda, 2006), which results in a nebula-captured hydrogen-rich protoatmosphere of the newborn planet. The amount of captured nebula gas depends mainly on

- nebular dissipation time and properties such as grain depletion factors,

- planetary growth rate and related protoplanet mass and size.

Disks are hydrodynamically stable, and magnetohydrodynamic turbulence, which is linearly unstable, plays a major 
role in disk physics. The nebula dissipation time is also related to the activity of the host star and the presence of massive stars and supernovae in the neighborhood of the particular system. The removal of the disk is related to efficient angular momentum transport processes, the $\mathrm{X}$-ray and extreme ultraviolet (EUV) radiation field, and the stellar plasma flow during the T-Tauri, post-T-Tauri, and activity saturation stage of the host star. Normal and transition disks show a wide gap of missing warm dust near the central star.

Dust in the disks is produced as a by-product of collisions between planetesimals and planetary embryos related to the planet-formation process. Planetary debris disks represent the near-final stage of the circumstellar disk evolution process; that is, they are the evolutionary products of ongoing or completed planet formation.

The general disk evolution scenarios are illustrated in Fig. 5. In protoplanetary disks, the inner radial boundary between magnetorotational instability turbulent (active) and magnetorotational instability quiescent (dead) zones plays an important role in some planet-formation scenarios and in disk evolution models. The extreme $\mathrm{X}$-rays, EUV, far ultraviolet (FUV), flux, and winds of the young active star clear the nebular gas in time. Inside the evolving disk are dust particles, a magnetically inactive dead zone, and the growing protoplanets. It should also be pointed out that many assumptions must be made mainly due to unknown dust properties and related opacities. These unknowns influence the results of each theoretical simulation. The discovered exoplanets move around in the disk and are not formed at the location where we observe them later. Further, as shown in Fig. 6, planets within a disk create torques and momentum exchange so that the semimajor axis also changes (Kley and Nelson, 2012). Tidal forces can also push the planets out of resonance. The disk acts as a damping process on eccentricity and inclination. If a planet becomes massive it opens up a gap in the disk, and if there are several planets they act on the disk and against each other. For example, numerical simulations of two massive gas giants in the system HD 73526, which adjoin a large gap in the disk, show that the outer planet can be pushed inward by the outer disk, while the inner planet is pushed outward by the inner disk (Sándor et al., 2007).

Many planets may also fall to the star. Migration may be too fast, but due to corotating torques the fast migration may slow down. Planets are captured into resonant systems that can be seen in observations so that it can be concluded that migration should occur. The data from exoplanets discovered by the Kepler satellite indicate that many systems are in (near) resonance, which indicates a resonant capture through a convergent migration process that is related to dissipation forces due to planet-disk interaction. Thus, the evidence for planet migration is confirmed due to the existence of

- resonant systems and flat Kepler systems

and

- hot Jupiters and Neptunes.

To overcome the related uncertainties in disk physics, such as migration in turbulent disks, self-gravitating disks, tidally driven migration, type-III migration, and stellar irradiation, it is very important and timely that theoreticians cooperate more with observers so that a powerful strategy for missing observables related to theoretical disk models can be established. For instance, the accretion luminosity could be high so that one should search for a protoplanet inside a disk (Kraus and Ireland, 2012). One main task for the future should therefore be

- to observe protoplanets in evolving disks.
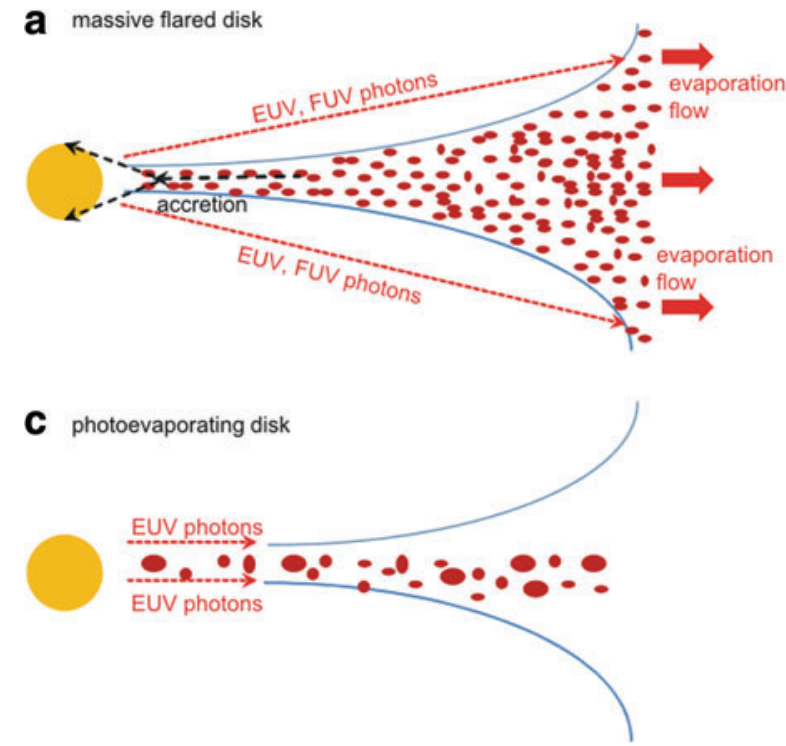

b settled disk

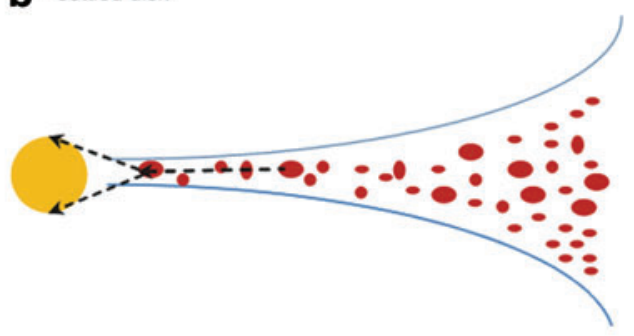

d debris disk

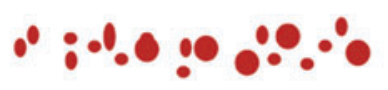

FIG. 5. Illustration of disk evolution and planet formation. (a) Strong X-rays, EUV, and FUV as well as winds interact with the gas/dust disk in its earliest stage. (b) Planetesimals and planetary embryos form due to collisions, the disk settles, and evaporation continues. Depending on the gravity potential of the embedded planetary embryos and protoplanets, nebula gas is attracted. (c) Photoevaporation continues, and planetary embryos and protoplanets that orbit near their host stars become decoupled from the evaporating nebula gas first. (d) After a few million years, but at the latest about $10 \mathrm{Myr}$, the debris disk remains, and planets may continue to form (after Williams and Cieza, 2011). Color images available online at www.liebertonline.com/ast 

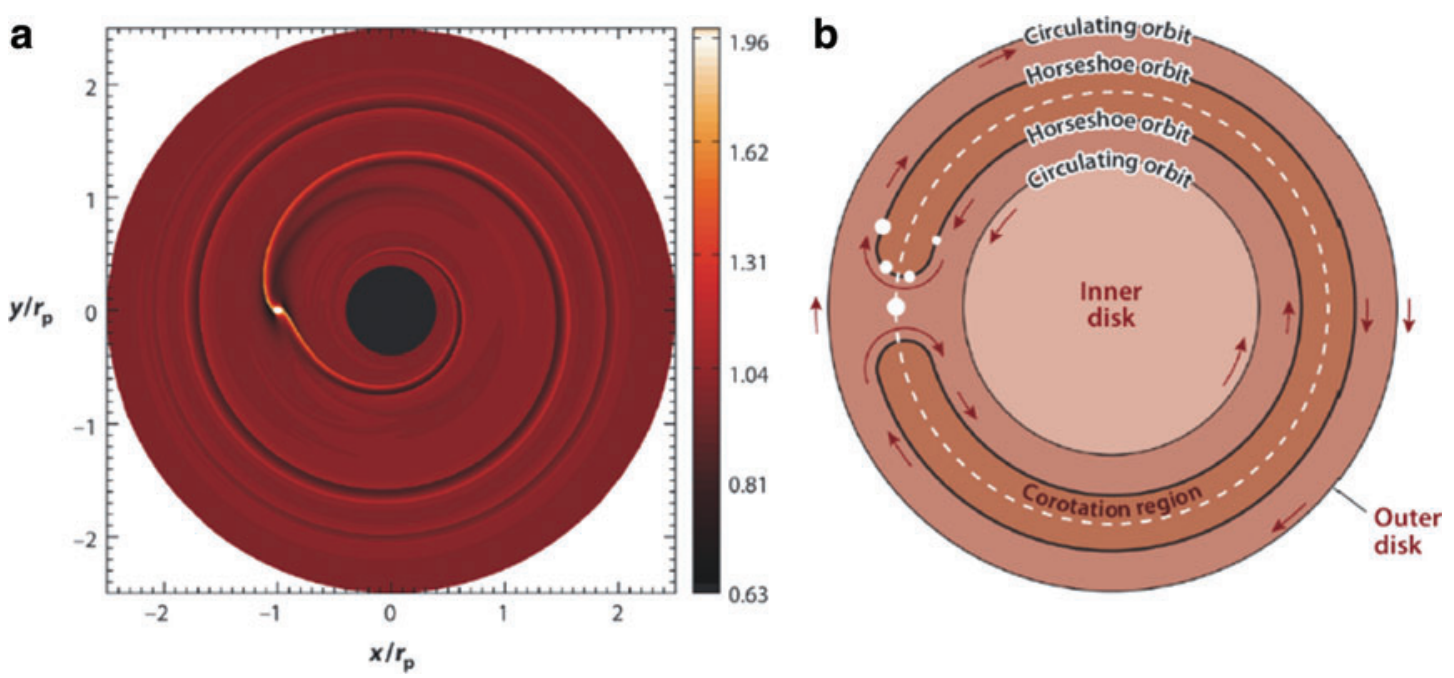

FIG. 6. Planet-disk interaction results in the following: (a) spiral arm formation that creates Lindblad torques on the planet, resulting in inward-moving planets; and (b) horseshoe regions that create corotation torques that can result in outwardmoving planets (from Kley and Nelson, 2012). Color images available online at www.liebertonline.com/ast

\subsection{Protoatmospheres and early water formation}

Another challenge related to the discovery and characterization of exoplanets and their structure, particularly in the rocky planet domain, is our capacity to study protoatmospheres and initial water inventories, and their formation and evolution. As illustrated in Fig. 7, the initial atmospheres and water content of terrestrial planets are related to a complex interplay between the nebula dissipation time, the growth rate/ time of a protoplanet from planetesimals and planetary embryos, the orbit location and water content of the initial building blocks (i.e., planetesimals and planetary embryos), outgassing processes from the interior, the impact history, and finally the host star's radiation and plasma environment.

The first atmosphere is created by the capture and accumulation of hydrogen, $\mathrm{He}$, and other gases from the

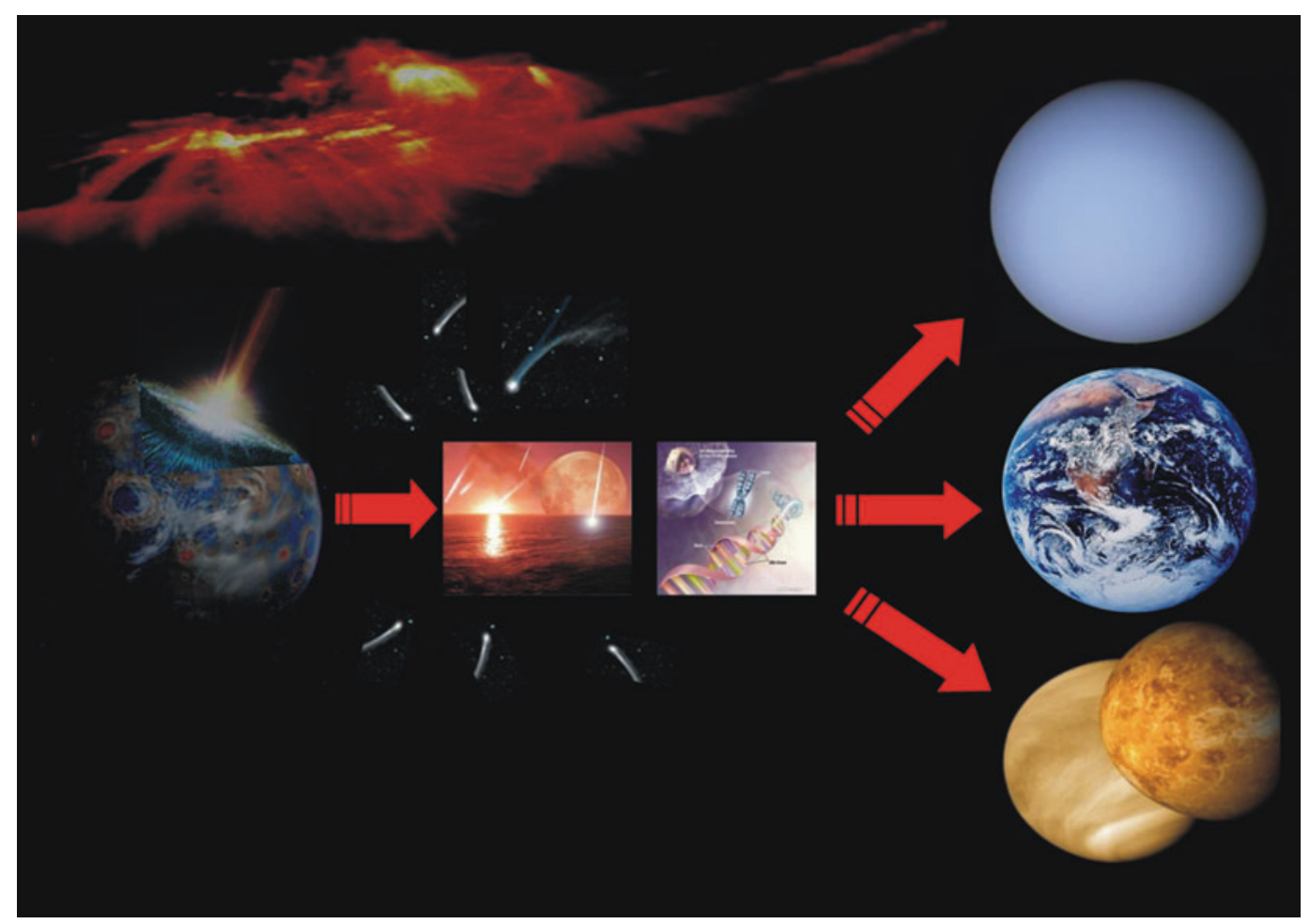

FIG. 7. Illustration of the evolution from protoplanets to various end products, which can be Venus- or Mars-like dry or frozen $\mathrm{CO}_{2}$-rich one-plate planets; geophysically active, water-rich Earth-like planets with continents and nitrogen atmospheres ( $\mathrm{CO}_{2}$ in carbonates); or a mini-Neptune or water world with a surrounding hydrogen corona (as seen in Figs. 2 and 5 ) in the event that the early planet accumulated too much nebula gas or originated with too much volatiles, which were not expelled by host star-powered atmospheric escape processes. Color images available online at www.liebertonline.com/ast 
surrounding disk in which the growing protoplanet resides (rocky core). Depending on the nebula properties, its lifetime, and the gravity potential of the growing and embedded protoplanet, an extensive amount of gas could be attracted so that optically thick, dense hydrogen envelopes accumulate around rocky cores (e.g., Hayashi et al., 1979; Mizuno, 1980; Wuchterl, 1993; Ikoma et al., 2000; Ikoma and Genda, 2006; Rafikov, 2006; Ikoma and Hori, 2012).

The recent discovery of low-density super-Earths with short orbital periods such as those in the Kepler-11 system indicates that nebula-captured gaseous envelopes may not so readily separate from fast-growing planets that are more massive than Earth or Venus (Ikoma and Hori, 2012; Lammer, 2013; Lammer et al., 2013). Preliminary results indicate that the atmospheres of massive rocky bodies undergo runaway disk gas accretion, while the atmospheres of light rocky bodies undergo significant erosion during disk dispersal. In atmospheric erosion, the heat content of the rocky body plays an important role (Ikoma and Genda, 2006; Ikoma and Hori, 2012).

As one can see, the formation of dense nebula-based hydrogen envelopes around super-Earths is closely linked to the open questions and future challenges discussed briefly in previous sections and shows that the structural characterization of low-density super-Earths with future space missions like CHEOPS, TESS, and PLATO provide important clues for understanding planetary accretion and disk evolution. The second atmosphere formation occurs by catastrophic outgassing of dense hot $\mathrm{H}_{2} \mathrm{O}, \mathrm{CO}_{2}, \mathrm{CH}_{4}, \mathrm{NH}_{3}$ atmospheres during the magma ocean solidification process when the planet's accretion ended. Earth- or super-Earthtype planets with silicate mantles and metallic cores will accumulate a major amount of their initial water and carbon compounds during the second atmosphere-formation process.

As illustrated in Fig. 8, modern terrestrial planet-formation model simulations indicate that terrestrial planets originate from differentiated planetesimals to large planetary embryos with sizes of several hundred to a few thousand kilometers (Kokubo and Ida, 2000; Raymond et al., 2004; Alibert et al., 2007; Raymond et al., 2009; Lunine et al., 2011; Walsh et al., 2011; Morbidelli et al., 2012). After the evapo- ration of the nebula gas due to the extreme EUV phase of the young Sun/star, the protoplanets continue to grow through the capture and collisions of remaining large planetesimals and planetary embryos. Impact studies related to Mercury and the Moon indicate that giant impacts between planetary embryos do not completely devolatilize a planet.

During huge impacts, a fraction of the volatiles remain inside the growing planetary body, while some volatiles are pushed outward and may escape into space.

Geochemical studies have revealed that a fraction of Earth's water originated from comets, while the majority came from chondritic rocky materials (Mumma and Charnley, 2011). Recent studies indicate that most of the water likely originates from rocky planetesimals (Alexander et al., 2012). Meteorites are therefore candidate building block materials for planetary embryos and protoplanets that have a wide range of carbon and water contents. Various degassing and formation scenarios during accretion for a wide range of atmospheric masses and composition of exoplanets within 1-30 $M_{\oplus}$ have been modeled (Elkins-Tanton and Seager, 2008; Elkins-Tanton, 2011).

By using primitive and differentiated meteorite compositions, these studies have revealed that degassing alone can produce a wide range of planetary atmospheres that range from $\leq 1 \%$ of a planet's mass up to $\sim 6 \%$ by mass of hydrogen, $\sim 20 \%$ by mass of $\mathrm{H}_{2} \mathrm{O}$, and $\sim 5 \%$ by mass of carbon compounds (Elkins-Tanton and Seager, 2008). Depending on the initial volatile contents and planetary building blocks, magma ocean outgassing models, as shown in Fig. 10, can produce early steam atmospheres with surface pressures up to several $10^{4}$ bar (Elkins-Tanton, 2011). It should be noted that hydrogen-rich atmospheres can also be formed by outgassing due to oxidation of metallic Fe with $\mathrm{H}_{2} \mathrm{O}$ and that atmospheric escape processes that are powered by the high radiation and plasma environment of the young host star are not included in the results shown in Fig. 9. Catastrophically outgassed steam atmospheres during the magma ocean solidification are expected to be related to the formation of the earliest oceans on Earth (e.g., Elkins-Tanton, 2008; Lammer et al., 2012; Hamano et al., 2013) or exoplanets within the habitable zone in general (e.g., Elkins-Tanton, 2011).

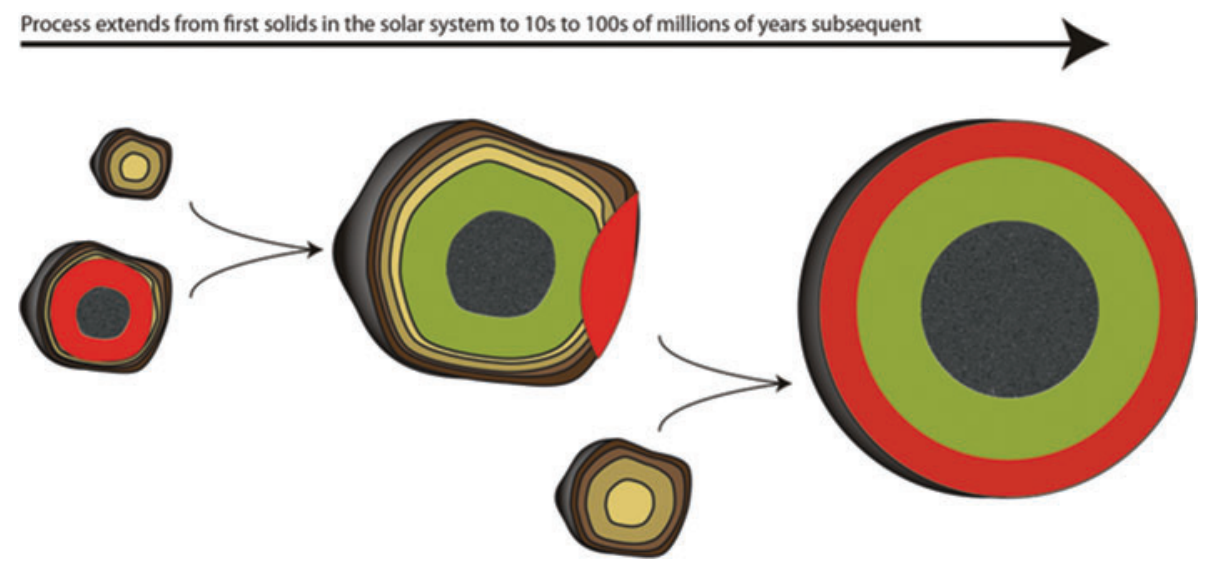

FIG. 8. Illustration of protoplanet growth and formation due to collisions between differentiated planetesimals and planetary embryos. The red areas correspond to magma, the other layers to mantle and core materials (courtesy of L. ElkinsTanton). Color images available online at www.liebertonline.com/ast 


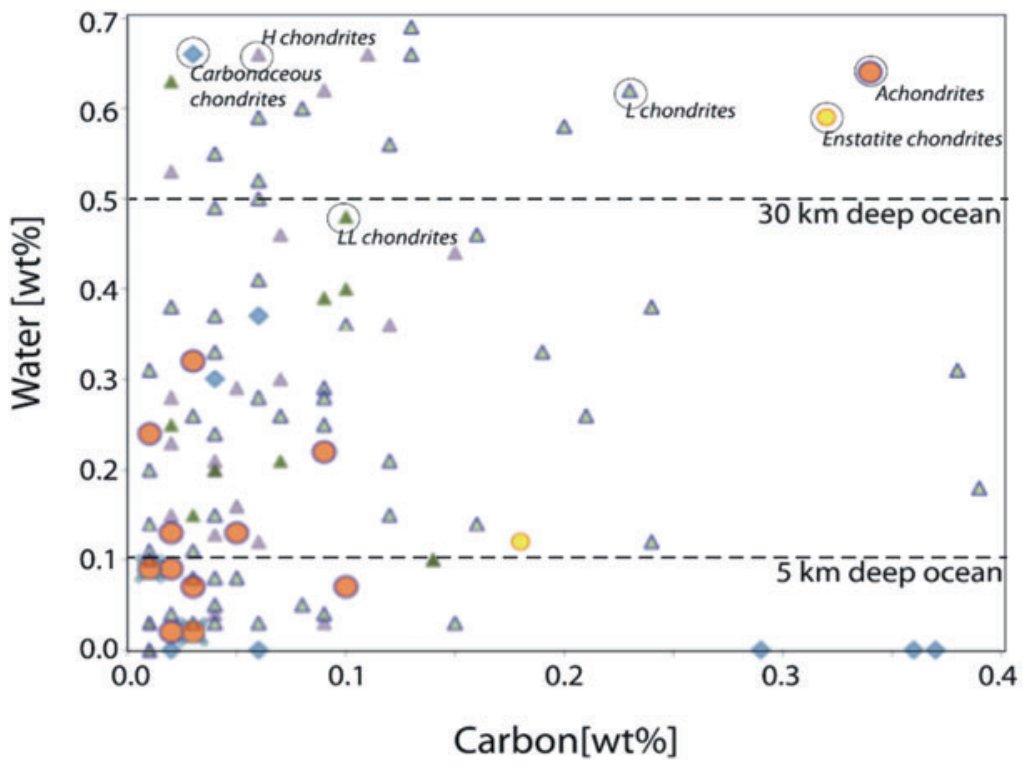

FIG. 9. Initial water and carbon contents in weight percent and resulting water ocean depths after condensation of an outgassed steam atmosphere. The range of water used in the figure includes meteorites that are likely building blocks for terrestrial planets (Jarosewich, 1990). The dashed lines show the resulting water ocean depth in kilometers produced by a collapsed degassed steam atmosphere of a planet related to the initial bulk mantle water content (courtesy of L. ElkinsTanton). Color images available online at www.liebertonline.com/ast
However, it should be noted that differentiated large planetary embryos as shown in Fig. 8 will also form magma oceans such that $\mathrm{H}_{2} \mathrm{O}$ and other volatiles may be stepwise outgassed and potentially lost to a great extent, as illustrated in Fig. 10, before the final magma ocean solidifies when the final planet's accretion ends. If wet planetary embryos lose much of their water inventories during their growth to the final planetary body, the volatile content that is outgassed in the final stage would be lower than expected. Such a scenario would agree with the hypothesis of Albarède and BlichertToft (2007), which is that the terrestrial planets in the Solar System accreted dry and obtained most of their water during the late veneer via impacts. On the other hand, the discoveries of $\mathrm{H}_{2} \mathrm{O}$ /volatile-rich super-Earths like GJ 1214b support the outgassing hypothesis as briefly discussed before. From the first discovery of lower mass and size exoplanets, it is clear that we cannot take the Solar System planets as a base for extrapolations, because they may only represent a particular outcome of a huge possibility of final planetary scenarios, though they may fit within the three overall categories illustrated in Fig. 7.
It is important not to forget atmosphere-producing processes that are related to secondary outgassed atmospheres of $\mathrm{H}_{2} \mathrm{O}, \mathrm{CO}_{2}, \mathrm{~N}_{2}$, and other trace gases via tectonic activities, volcanoes, and impact events such as the Late Heavy Bombardment in the Solar System. Most likely, terrestrial planets may obtain their initial atmospheres by a mixture of all the above-discussed scenarios in one or more ways.

The participants of the ISSI forum were convinced that detailed research related to the latest and near-future discoveries of Earth- and super-Earth-like exoplanets and their characterization in structure and atmosphere, together with the expertise and knowledge obtained within the Solar System and geochemistry communities, are highly timely and necessary to understand how terrestrial planets obtain and lose their initial water inventories and volatiles. These are crucial questions related to habitability aspects in general, and from the discussion within the ISSI forum panelists, it can be understood that several linked and interacting processes related to disk properties, nebula dissipation time, planetary growth rate, host star powered atmospheric escape
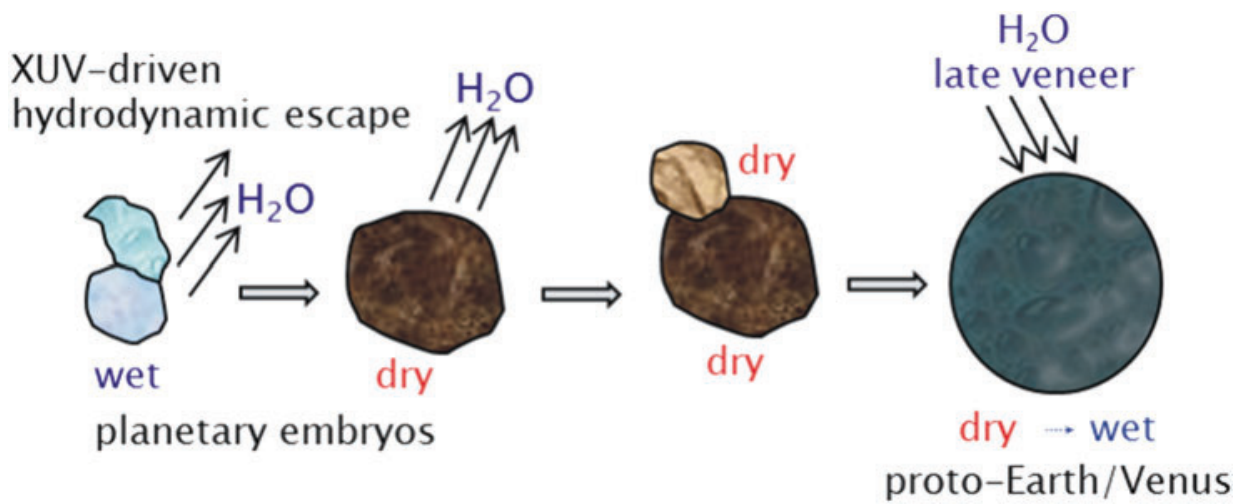

FIG. 10. Illustration of a late growth scenario that could be expected for Venus and Earth. Mars-sized planetary embryos will lose outgassed volatiles and their initial water inventories during collisions and growth to larger protoplanets. Finally, not much $\mathrm{H}_{2} \mathrm{O}$ would remain in the final planet, and oceans would have to be produced by later impacts of water-rich material. Color images available online at www.liebertonline.com/ast 
processes, and the general impact history of a particular system play important roles.

\section{Stellar Environments and Exoplanet Host Stars}

The long time evolution of planets and their atmospheres can only be understood if the evolution of the planet host stars are considered. Planets cannot be seen as isolated objects because the radiation and plasma environment of their host stars constitute a permanent forcing of the upper atmospheremagnetosphere environments of the exposed bodies. The main effect of these forcing factors is to ionize, chemically modify, heat, expand, evaporate, and erode the upper atmosphere throughout the lifetime of a planet (e.g., Lammer, 2013). The host star and its related activity influence the detection ability and make the measuring of exoplanetary parameters such as mass and radius more difficult. Physical and fundamental properties for exoplanet host stars include the star's mass, radius, chemical composition, and rotation period, which is connected to magnetic activity and age. These properties can be determined by the following methods:

- calibrations (from binaries, clusters, etc.);

- stellar evolution models;

- asteroseismology;

- age determination: kinematics, asteroseismology, activity, chemical abundances ( $\mathrm{Li}, \mathrm{Be})$, theoretical models.

The radiative properties can be studied if a detailed spectral energy distribution from X-rays to IR, and radio, was obtained. Short-term variations up to years can be studied by establishing time series with photometry. For longer timescales, observations of stellar proxies or stars of the same spectral class with different ages are the best options. Because the host star's activity evolution is different for different stellar spectral types, and furthermore depends on the stellar age and the location of the habitable zone around the host star, it can be expected that the atmospheric evolution of exoplanets is strongly coupled with the activity evolution [X-rays, EUV, FUV, winds, coronal mass ejections (CMEs), solar energetic particles, etc.] of the host star.

\subsection{The relevance of the stellar Lyman- $\alpha$ emission line observation and reconstruction methods}

For the characterization of exoplanetary atmospheres as well as the observation/detection of stellar mass loss, it is important to measure the Lyman- $\alpha$ emission line of the particular star. Accurate investigations of the chemical composition of exoplanetary atmospheres can only be carried out if the near-UV $(\lambda=170-320 \mathrm{~nm})$, FUV $(\lambda=117-$ $170 \mathrm{~nm}), \operatorname{EUV}(\lambda=30-91.1 \mathrm{~nm}), \operatorname{XUV}(\lambda=10-30 \mathrm{~nm})$, and $X$-ray $(\lambda<10 \mathrm{~nm})$ radiation from the host star, all of which control important molecular photodissociation and photoionization processes, are known. The Lyman- $\alpha$ emission line dominates the FUV spectra of late-type stars and is a major source for the photodissociation of important atmospheric molecules such $\mathrm{H}_{2} \mathrm{O}, \mathrm{CH}_{4}, \mathrm{NH}_{3}, \mathrm{CO}_{2}$. For the Sun, the Lyman$\alpha$ emission is $\sim 20 \%$ of the total flux between 1 and $170 \mathrm{~nm}$. For example, the solar Lyman- $\alpha$ line is responsible for $\sim 50 \%$ of the photodissociation rate of $\mathrm{H}_{2} \mathrm{O}$ molecules between 114.8 and $194 \mathrm{~nm}$ and $\sim 70 \%$ of the total photodissociation rate of $\mathrm{CH}_{4}$ between 5.6 and $152 \mathrm{~nm}$. The Lyman- $\alpha$ line emission is even more important for cooler low-mass stars, such as $\mathrm{M}$ dwarfs, because the photospheric emission at $\lambda>170 \mathrm{~nm}$ decreases rapidly with decreasing effective temperature. A comparison between the quiet spectrum of the Sun and that of GJ 876, which has an exoplanet within its habitable zone, indicates that, as would be discerned from an exoplanet in the habitable zone, the near UV is weaker by 4 orders of magnitude but the FUV is much stronger compared to that of the Sun. For GJ 876, the Lyman- $\alpha$ flux is about 2 times as high as seen from the star's habitable zone compared to the Sun as seen from the Earth. $M$ stars seem to be brighter in Lyman- $\alpha$ compared to more massive Sun-like stars, and the age of the stars, which is related to their activity in general, also plays a major role (France et al., 2013; Linsky et al., 2013).

Exoplanetary atmospheres absorb the Lyman- $\alpha$ flux from their host stars without attenuation; neutral hydrogen in the interstellar medium (ISM) scatters most of the Lyman- $\alpha$ flux out of the line of sight between the star-exoplanet system and Earth. Because of this, the Lyman- $\alpha$ emission line cannot be directly measured because neutral hydrogen in the ISM attenuates the majority of the flux before it reaches Earth (Linsky et al., 2013). Therefore, it is important to reconstruct the stellar Lyman- $\alpha$ profiles and flux by correcting the ISM absorption.

To date, a reconstruction of the intrinsic Lyman- $\alpha$ line has only been carried out for a limited number of nearby stars that are bright enough, but this is not feasible for distant or faint host stars. Recently, Linsky et al. (2013) identified five methods for the reconstruction of the intrinsic Lyman- $\alpha$ flux from main-sequence stars between spectral types F5 and M5. From this study, it is shown that the quality of the data determines which reconstruction method yields the most accurate result.

The most accurate method for the reconstruction of stellar Lyman- $\alpha$ emission was developed by Wood et al. (2005) and requires high-resolution spectra of the Lyman- $\alpha$ line and knowledge of the interstellar velocity structure based on high-resolution spectra of the deuterium Lyman- $\alpha$ and metal lines. The errors from such reconstructed profiles are at $\sim 20 \%$. At present, only the STIS instrument on HST can provide such data for nearby stars. Another method developed by France et al. (2012) also requires high-resolution spectra of the hydrogen Lyman- $\alpha$ emission line but does not require spectra of the deuterium Lyman- $\alpha$ line or any other interstellar absorption line. It should be noted that this particular reconstruction method fails when the interstellar velocity structure has many components, which is unknown in the absence of high-resolution interstellar absorption lines. A third method described by Linsky et al. (2013) requires flux measurements of the stellar C IV, C II, O I, Mg II, or Ca II lines and the best-fit correlations of these lines with fluxes of the reconstructed Lyman- $\alpha$ lines in existing data sets. The accuracy of Lyman- $\alpha$ flux estimates for F5-K5 dwarf stars by this method is about $\sim 18-25 \%$. To obtain these accuracy values, high-resolution spectra of the $\mathrm{Mg}$ II lines for interstellar absorption must be corrected. This particular method is based on the hypothesis that the ratios of the Lyman- $\alpha$ line flux to C IV, C II, O I, Mg II, and Ca II line fluxes for stars of similar spectral type depend only gradually on line flux. The method does not work well for M0-MV dwarf stars. For these stars, the dispersions are larger because of the stellar variability between the observing times of Lyman- $\alpha$ and the other lines (Linsky et al., 2013). The largest uncertainty here is 
the stellar metal abundance; therefore more observations of M-type stars will be needed to better understand the uncertainties associated with low metal abundances.

If there are no UV or Ca II line flux observations available, but X-ray measurements with an energy range similar to ROSAT observations have been made, a method can provide estimates of the intrinsic Lyman- $\alpha$ flux from least-squares fits to the intrinsic Lyman- $\alpha / X$-ray flux ratio versus the X-ray flux. The average dispersions about the fit lines for F5-G9 and $\mathrm{K} 0-\mathrm{K} 5$ stars are of the order of $\sim 20-30 \%$. However, this method should not be applied to $M$ stars, which show a much larger dispersion because of the large time variability of X-rays and the comparison of X-ray and Lyman- $\alpha$ data obtained at different times. When no Lyman- $\alpha$, UV emission lines, or X-ray data are available from observations, the Lyman- $\alpha$ flux for F5-M5 stars can be reconstructed based only on the stellar effective temperature and stellar activity measurements, and stellar parameters within an accuracy of 30 40\% (Linsky et al., 2013).

\subsection{Radiation and plasma impact on planetary atmospheres and habitability}

Activity in late-type stars (i.e., spectral types G, K, M) has been intensively studied in the past decades with satellites such as ASCA, ROSAT, Chandra, XMM-Newton, EUVE, FUSE, and IUE. In particular, the radiation environment of Sun-like G stars has been studied for several so-called solar proxies with ages from $\sim 100$ Myr to $\sim 8.5$ Gyr with high accuracy within the "Sun in Time" project (Dorren and Guinan, 1994; Dorren et al., 1995; Güdel et al., 1997; Ribas et al., 2005; Claire et al., 2012). From these data, it can be concluded that the integrated flux between 0.1 and $120 \mathrm{~nm}$ was $\sim 100$ times more intense than that of today's Sun during the first $100 \mathrm{Myr}$ after its arrival at the zero-age main sequence (ZAMS) (Ribas et al., 2005; Claire et al., 2012), before it decreased according to a power law to a factor of $6, \sim 3.5$ Gyr ago. This activity-age relation indicates that lower-mass M-type stars spend more time in this highly active, but saturated, phase before their activity begins to decrease. It has been found that low-mass $\mathrm{M}$ dwarfs have saturated emission periods up to 0.5-1 Gyr and possibly longer for late-type M stars (Scalo et al., 2007).

Figure 11 shows the X-ray to bolometric luminosity for all stars with masses $<0.9 M_{\odot}$, which are given in the ROSAT catalogue with distances that have been measured by Hipparcos (Scalo et al., 2007). Because the X-ray luminosities are given in relation to the total stellar luminosity, they correspond also to the X-ray flux that exposes a planetary upper atmosphere within the habitable zone of the particular star. The X-ray/bolometric luminosity value for the present Sun is given by the tiny black dot on the bottom of the right $y$ axis. From these observations, it can be concluded that for an average dMe star, the steady coronal X-ray flux above the atmosphere of a habitable planet could be about a thousand times larger than that of present-day Earth, while during the largest flares this flux could be a million times larger.

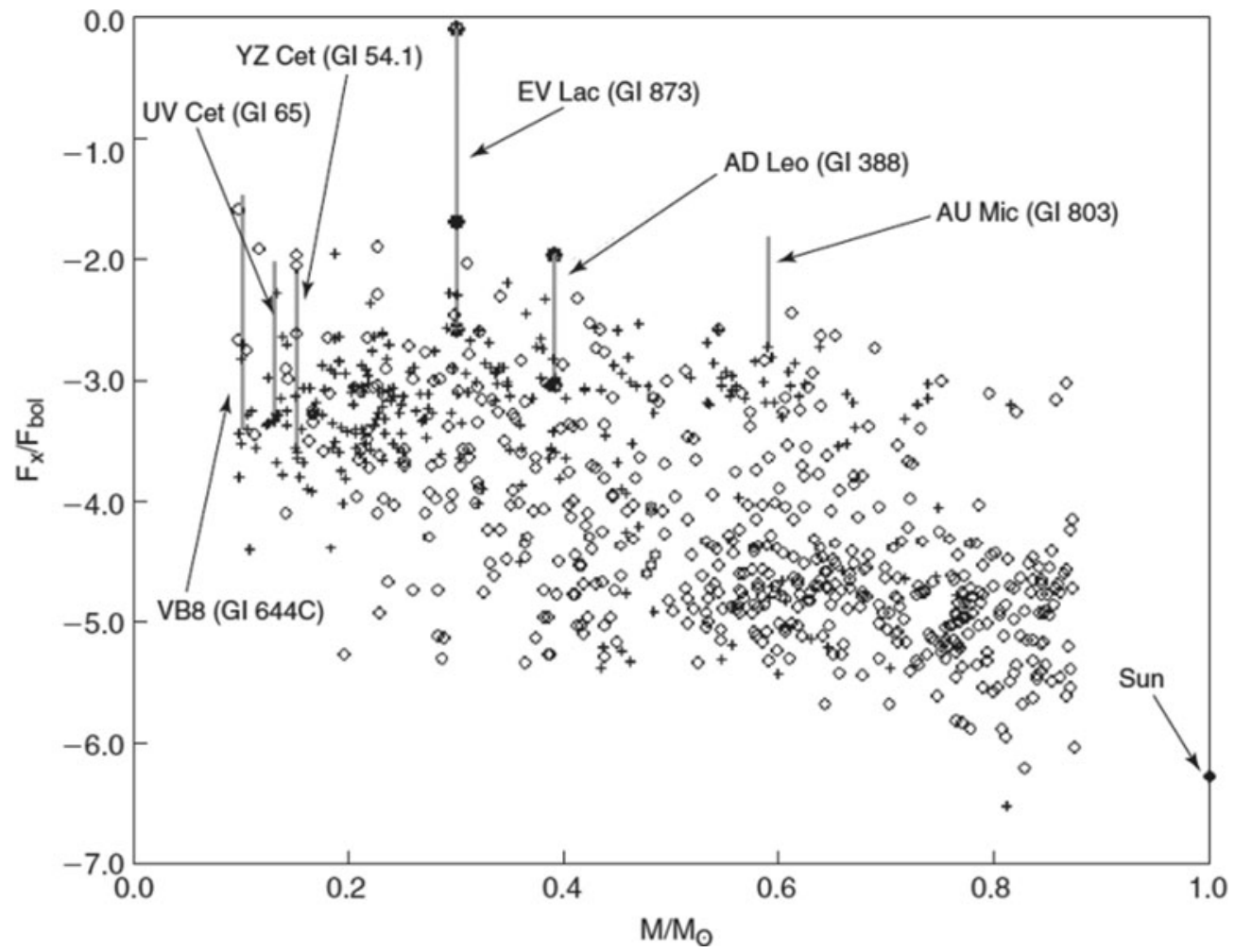

FIG. 11. Scatterplot of the ratio of soft X-ray to bolometric luminosity as a function of stellar mass, for every star with mass $<0.9 M_{\odot}$ in the ROSAT catalogue within Hipparcos distances, compared to the present Sun. The vertical gray lines show the amplitude variations of very large X-ray flares of well-studied $\mathrm{M}$ stars. The X-ray luminosities are given relative to the star's total luminosity so that they also represent the fraction of flux above the atmosphere of any planet in a particular habitable zone (after Scalo et al., 2007). 
The response of the upper atmosphere of an Earth-like exoplanet to such high short-wavelength radiation levels would be tremendous. As shown by Tian et al. (2008a, 2008b) and Lichtenegger et al. (2010), an Earth-like $\mathrm{N}_{2}$-dominated thermosphere, like hydrogen-rich atmospheres, would experience a rapid transition to a hydrodynamic expansion regime if it is exposed to XUV flux values $\geq 7$ times that of today's Sun.

Because nitrogen is not a strong IR cooler, the upper atmosphere will move above expected magnetopause standoff distances so that the neutral constituents beyond the magnetopause could be ionized and picked up by the stellar wind. The stellar plasma can lead to efficient nonthermal atmospheric loss processes especially at close-in exoplanets (i.e., terrestrial planets within low-mass $M$ star habitable zones), which are exposed to dense plasma flows close to their host stars. During the past decade, several indirect methods for the study of stellar winds of solar-type stars have been developed. The method that, to date, has been most successful is the search for astrosphere absorption in the region where the stellar wind plasma collides with the local ISM and energetic neutral hydrogen atoms are produced via charge exchange. From the rise in absorption on the blue side of the stellar Lyman- $\alpha$ line, the mass loss rate (Wood et al., 2002, 2005) can be estimated. Mass loss rates of about a dozen Sun-like stars and cooler stars have been derived by this method from observations with the HST/STIS spectrograph. Depending on the age of the observed star, the mass loss rates are in the order of $<0.2-100$ times the present solar rate. From these mass loss rates, the stellar wind density and velocity at orbital distance of a planet can be estimated (Grießmeier et al., 2004). The corresponding estimates for the stellar wind of a 500 Myr old Sun-like star yield a factor 30-200 denser wind at 1 AU, which is about twice as fast as that of the Sun at present (Lichtenegger et al., 2010). Unfortunately, there are currently no accurate stellar mass loss observations for main-sequence stars that are younger than 0.7 Gyr. However, there is evidence of a possibly preferred appearance of CMEs in polar regions of rapidly rotating young stars/Sun (Strassmeier, 2009), which can lead to the propagation of shocks and magnetic ropes off the ecliptic plane during this early period (Zaqarashvili et al., 2011). These are crucial questions that need to be answered in order to understand the influence of winds and plasma outflows of very young stars after their arrival at the ZAMS. Upper limits to mass loss rates of young Sun-like stars may also be detected directly during the near future with ALMA by measuring the millimeter-wave bremsstrahlung flux emitted by the ionized stellar wind. This method becomes possible for the first time at millimeter wavelengths, given ALMA's unprecedented sensitivity.

A stellar/solar wind that is 30 times denser and 2 times faster than the present proton outflow of our Sun is able to erode a 1 bar $N_{2}$ atmosphere that is exposed to a 20 times higher XUV flux compared to that of the present Sun from a planet of Earth size and mass at $1 \mathrm{AU}$ during $10 \mathrm{Myr}$ (Lichtenegger et al., 2010). Because the XUV flux within M star habitable zones remains at high levels for much longer times than for G stars (see Fig. 11), it is unlikely that Earthlike habitats with nitrogen-dominated atmospheres can evolve under such extreme environments (Lammer et al., 2009, 2012; Lichtenegger et al., 2010), while mini-Neptunes, extreme water worlds with hydrogen envelopes, and superEarths with massive $\mathrm{CO}_{2}$-rich atmospheres will be more resistant against the high XUV radiation.

Lower-mass planets that are exposed to such high radiation values may lose their atmospheres and most likely become Mercury- or Mars-type bodies. Thus, from the stellar radiation point of view, Earth-like planets with stable nitrogen-rich atmospheres may be much more numerous within habitable zones of $G$ stars compared to lower-mass dwarf stars. Furthermore, the detection of stellar winds and related mass loss from young stars would also enhance our understanding of the evolution of stellar mass and its related luminosity. A better understanding of the mass and luminosity evolution of young stars is directly linked to the so-called Faint Young Sun Paradox, which touches on the fundamental question on paleoclimate and the origin of life on Earth and is therefore in the focus of present-day astrobiology research.

Another important point related to stellar forcing of upper atmospheres is the production of abiotic oxygen, which could have important consequences for future spectroscopic biosignature searches in exoplanetary atmospheres. For example, a super-Earth near the inner boundary of the system's habitable zone will outgas a dense steam atmosphere, the result of which would be surface pressures of $10^{4}$ bar (ElkinsTanton, 2008, 2011); thus, for such dense steam atmospheres, preliminary atmospheric escape studies indicate that a huge fraction of a few Earth ocean equivalent amounts of oxygen would result from the dissociation of $\mathrm{H}_{2} \mathrm{O}$ molecules by the host star's XUV radiation and likely remain in the upper atmosphere.

Because it is very unlikely that several Earth ocean equivalent amounts of oxygen would be lost by nonthermal atmospheric escape processes or oxidized at the planet's surface, it can be expected that there are exoplanets where this remaining oxygen forms an ozone layer of abiotic origin in the atmosphere. Such results agree with previous suggestions of Kasting (1995) and Chassefière (1996) that there may be planets, depending on their size, mass, orbital distance, as well as their host star's XUV flux evolution, that could accumulate huge amounts of abiotic oxygen. In the worst case related to the search for biosignatures in exoplanetary atmospheres, a planet could be observed that has $\mathrm{H}_{2} \mathrm{O}$ and a dense abiotic $\mathrm{O}_{3}$ layer in its atmosphere. Thus, the probability that such planets and atmospheres may exist raises the possibility of future false-positive detections of atmospheric biosignatures.

From the intense discussions related to the various issues briefly summarized before the ISSI forum, participants pointed out that the characterization of exoplanetary atmospheres can only be done accurately if the host star's chemical and physical parameters and evolutionary stage are also known to a great extent. Moreover, for understanding the evolution of exoplanets and their structure, the host star parameters over their age should also be known. To understand how planetary atmospheres and their water inventories can survive the extreme X-ray and EUV activity of their host stars after they arrive at the ZAMS (Fig. 12), thermal and nonthermal upper atmospheric processes must be examined by the development of self-consistent, multi-species ionospheric-thermospheric-exospheric models for the study of hydrostatic and nonhydrostatic dynamically expanding 


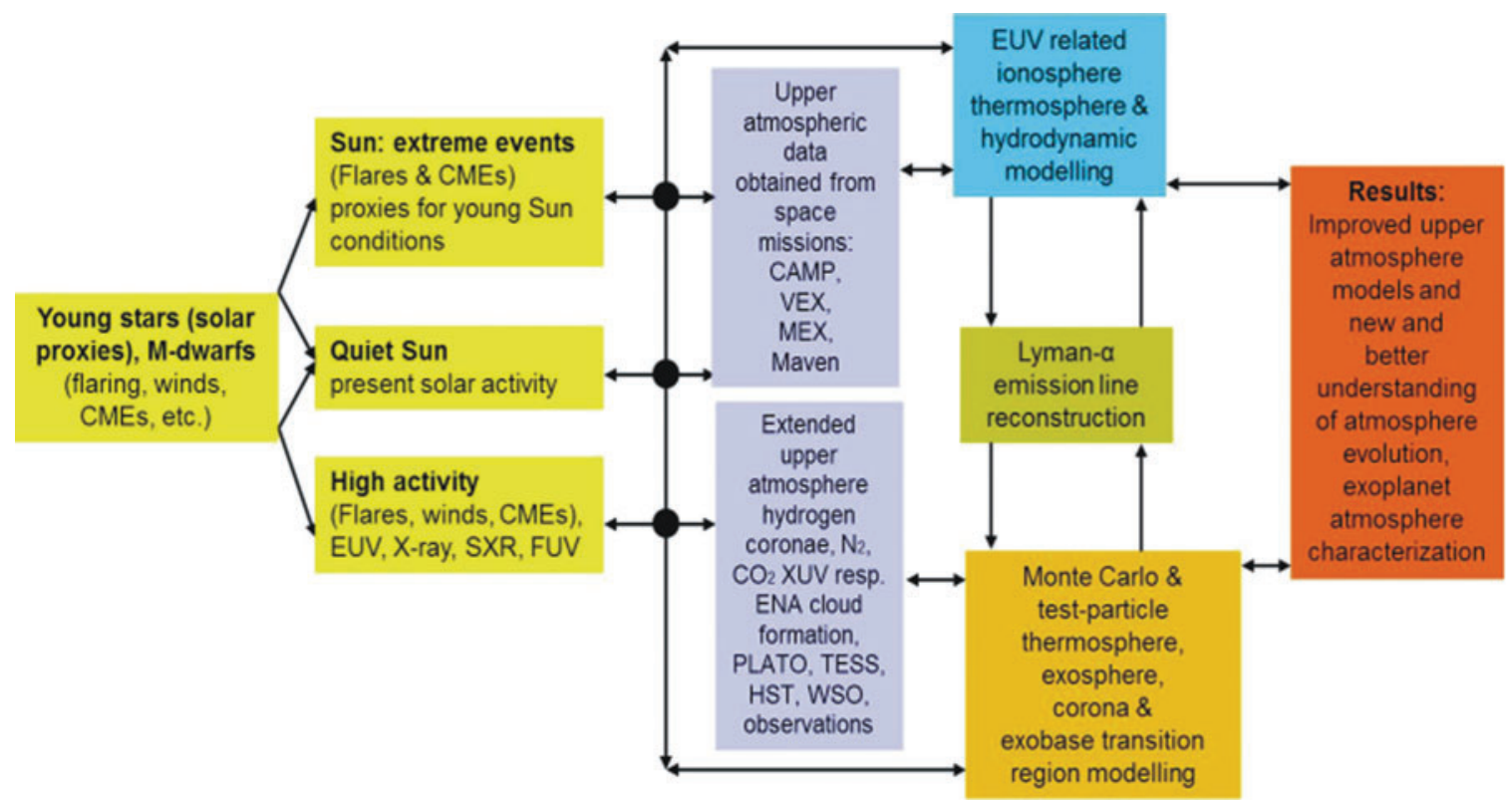

FIG. 12. Illustration chart about the general approach and connections between stellar/solar environment observations and modeling efforts within Solar System and exoplanetary research, which should lead to the development of state-of-the-art exoplanetary atmosphere-thermosphere-exosphere models and a better understanding of aeronomically related atmospheric evolution and habitability aspects for terrestrial exoplanets in general. Color images available online at www.liebertonline.com/ast

thermosphere-exosphere regions, which would include investigation of the photochemical production of exothermal "hot" atoms, their collisional interaction and transport within highly XUV-exposed thermospheres, and the formation of energetic neutral atoms (or ENAs).

\section{Future Outlook}

Given the intensive discussions during this ISSI forum, it became clear that exoplanetary science and discoveries have strong links to Solar System research, but the planets in the Solar System cannot be a blueprint for exoplanets, because each system and its evolving planets have a unique history that results in various end products. On the other hand, the different planets in the Solar System are the only examples that can be studied in situ, and they should be used as test cases in exoplanetary studies. In the Solar System sciences, important data, analysis, and sophisticated numerical models have evolved over the past few decades, and at this juncture Solar System research needs to be merged with the expanding field of exoplanetary planetology in a comparative way.

For instance, coordinated studies on the behavior of the upper atmospheres; ionospheres; magnetosphere environments; and thermal and nonthermal atmospheric loss processes on Venus, Earth, and Mars during extreme solar events [i.e., enhanced solar EUV and X-ray radiation, neutron fluxes, CMEs, intense energetic solar proton/electron fluxes (e.g., solar energetic particles), auroral phenomena, magnetic storms] can be used as proxies for enhancing our knowledge of particular exoplanetary atmospheres within extreme stellar environments such as M star habitable zones. The same studies would also serve as a proxy for the influence of the active young Sun with implications for the evolution of planetary atmospheres of younger Solar System planets.
Other areas that have important implications for the fastevolving field of exoplanetary science and Solar System research are related to early systems, planet, and protoatmosphere formation as well as the origin and delivery of water to planetary bodies. The discovery of small transiting exoplanets within the size and mass domains of Earth- to Neptune-type bodies at various orbital distances will allow us to define a realistic radius-mass boundary for Earth-like planets at a time when they can lose their protoatmospheres such that they can evolve to Earth-type habitats rather than to mini-Neptunes or hydrogen/volatile-rich water worlds. Because the origin and loss of the protoatmospheres depend on the activity of the stellar spectral type, the related disk physics and nebula evaporation time, and planetary growth rate, it can be expected that a radius-mass boundary for Earth-like planets will be determined as it relates to host star type. The origin, delivery, and loss of water during the formation of planets from planetesimals via planetary embryos to protoplanets were also identified as a hot and very important science case. This field is highly interdisciplinary and involves studies related to planet formation; meteorite, asteroid, and comet research; geochemical studies related to magma oceans; outgassing; impact research; stellar/solar physics; and aeronomy in relation to water escape from small and Mars-sized bodies.

Finally, all previously recommended research activities should be linked in an interdisciplinary manner to further our understanding of habitability and the evolution of planets within habitable zones toward potential habitats of various kinds. How many of these huge numbers of different planetary end products could evolve to become habitable planets where life might originate will certainly be one of the major research endeavors over the course of the next few decades. Because the mass of Earth-sized planets and superEarths within close-in $M$ star habitable zones can be 
determined more easily compared to an Earth in a Sun-like system at $1 \mathrm{AU}$, and because $\mathrm{M}$ stars are the most numerous spectral type, the habitability of dwarf star planets is currently highly debated. Recent estimates based on the Kepler survey indicate that there are numerous dwarf stars with terrestrial planets within their habitable zones in our Galaxy (e.g., Dressing and Charbonneau, 2013).

However, due to the host stars' emitted long-lasting short wavelength radiation (X-rays, EUV), the near location of the habitable zone (dense stellar winds, CMEs), and several geophysical differences (tidal-locking, climate, etc.) compared to Earth-like planets in orbits within habitable zones of Sun-like stars, possible exotic habitats may be expected to occur, though planets that are more similar to Earth will certainly be rare in such extreme stellar environments (Scalo et al., 2007; Lammer et al., 2009, 2012). Besides M star planet ground-based research projects such as MEarth, the TESS space observatory and planned future characterization missions such as EChO, including the JWST, will be tools for the discovery and characterization of $\mathrm{M}$ star terrestrial planets/ exotic habitats. For understanding whether Earth-like habitats that are geophysically active during billion-year periods with liquid water and continents on their surface are common or rare, terrestrial exoplanets from Earth size to superEarth size within G star habitable zones around 1 AU will need to be discovered. For such targets, the precision for the Doppler method must be pushed down from the present $5 \mathrm{~m} \mathrm{~s}^{-1}$ to $\sim 1 \mathrm{~m} \mathrm{~s}^{-1}$ so that the masses of the discovered planets could be measured, while the transit search for Earthsized planets/habitats in orbits of bright nearby Sun-like stars by a mission like PLATO and follow-up characterization projects could be carried out. From the discussions during the ISSI forum, the following recommendations and outcome can be summarized as

- more exoplanet detections around bright and nearby stars are essential if we want to characterize exoplanetary atmospheres more accurately and to a greater extent than they presently are.

Because no other mission or project other than the proposed PLATO and TESS missions will fill the technologyrelated lack of discoveries of exoplanets around bright nearby stars, from those that reside in close-in habitable zones (TESS) to those that reside in Solar System-like habitable zones at 1 AU (PLATO), the forum participants agreed that these space observatories are necessary to ensure that planned future exoplanetary atmosphere characterization missions can fulfill their proposed science cases. From the observational side, the forum participants agreed it is very important that

- the accuracy of the exoplanet mass determination with the Doppler method should be pushed down from the present-day precision of $0.5 \mathrm{~m} \mathrm{~s}^{-1}$ to a precision of $0.1 \mathrm{~m}$ $\mathrm{s}^{-1}$ so that the masses of Earth-like planets at orbital distances up to the habitable zone around a Sun-like star at $1 \mathrm{AU}$ can be measured.

- a large number of exoplanets from Earth size to Jupiter size should be sought around bright and nearby stars for characterization of exoplanetary atmospheres and their systems.

- the present time error bars related to planet size (and mass, see first point) have to be minimized by ultrahigh- precision photometry, and so on, such that more accurate mean densities and resulting bulk compositions of exoplanets can be discerned. This is very relevant because the bulk composition that could, in theory, be determined from precise mass-radius relations is directly linked to the planet's interior and is necessary for the characterization of new planet categories such as super-Earths (exo-Mercuries), water worlds, and miniNeptunes (see Figs. 1 and 4). Because we do not know what kind of material comprises the interior of Jupiter in the Solar System, we may never acquire detailed information on the inner structure of an exoplanet. However, together with precise radius-mass measurements and transmission spectroscopy during transits, dense gaseous layers or primordial gas captured from the nebula during a planet's early formation could be identified. As discussed above for GJ 1214b, these combined methods may even reveal information related roughly to the core properties (i.e., dense rocky core, low-density core, icy core, and deep water layer).

- the age of planetary systems should be better constrained, for example by asteroseismology, to study potential evolutionary aspects.

Furthermore, the forum participants advised that ISSI may support workshops during the next 2 years that will strongly benefit the above-mentioned research areas. The identified timely workshop topics proposed to ISSI during $2013 / 2014$ by the international research community should be related to

- the origin and formation of protoplanets and the delivery of water from planetary embryos to protoplanets;

- the disk in relation to the formation of planets and their protoatmospheres in terms of efforts to constrain the formation from observations;

and

- star-planet atmosphere interaction versus stellar distance and evolutionary consequences (spectral type: M star habitable zone planets, chemistry, magnetosphere, etc.).

\section{Abbreviations}

CHEOPS, CHaractizing ExOPlanets Satellite.

CMEs, coronal mass ejections.

CoRoT, Convection, Rotation and planetary Transits.

EChO, Exoplanet Characterisation Observatory.

ELT, Extreme Large Telescope.

ENAs, energetic neutral atoms.

EUV, extreme ultraviolet, $\lambda=30-91.1 \mathrm{~nm}$.

FUV, far ultraviolet, $\lambda=117-170 \mathrm{~nm}$.

HARPS, High Accuracy Radial Velocity Planet Searcher.

HST, Hubble Space Telescope.

ISM, interstellar medium.

ISSI, International Space Science Institute.

JWST, James Webb Space Telescope.

PLATO, PLAnetary Transits and Oscillations of stars.

STIS, Space Telescope Imaging Spectrograph.

TESS, Transiting Exoplanet Survey Satellite.

XUV, extreme ultraviolet, $\lambda=10-30 \mathrm{~nm}$.

ZAMS, zero-age main sequence. 


\section{References}

Adams, E.R., Seager, S., and Elkins-Tanton, L. (2008) Ocean planet or thick atmosphere: on the mass-radius relationship for solid exoplanets with massive atmospheres. Astrophys $J$ 673:1160-1164.

Albarède, F. and Blichert-Toft, J. (2007) The split fate of the early Earth, Mars, Venus, and Moon. Comptes Rendus Geoscience 339:917-927.

Albrecht, S., Winn, J.N., Johnson, J.A., Howard, A.W., Marcy, G.W., Butler, R.P., Arriagada, P., Crane, J.D., Shectman, S.A., Thompson, I.B., Hirano, T., Bakos, G., and Hartman, J.D. (2012) Obliquities of Hot Jupiter host stars: evidence for tidal interactions and primordial misalignments. Astrophys J 757, doi:10.1088/0004-637X/757/1/18.

Alexander, C.M.O'D., Bowden, R., Fogel, M.L., Howard, K.T., Herd, C.D.K., and Nittler, N.R. (2012) The provenances of asteroids, and their contributions to the volatile inventories of the terrestrial planets. Science 337:721-723.

Alibert, Y., Broeg, C., Benz, W., Wuchterl, G., Grasset, O., Sotin, C., Eiroa, C., Henning, T., Herbst, T., Kaltenegger, L., Léger, A., Liseau, R., Lammer, H., Beichman, C., Danchi, W., Fridlund, M., Lunine, J., Paresce, F., Penny, A., Quirrenbach, A., Röttgering, H., Selsis, F., Schneider, J., Stam, D., Tinetti, G., and White, G.J. (2007) Origin and formation of planetary systems. Astrobiology 10:19-32.

Batalha, N.M., Rowe, J.F., Bryson, S.T., Barclay, T., Burke, C.J., Caldwell, D.A., Christiansen, J.L., Mullally, F., Thompson, S.E., Brown, T.M., Dupree, A.K., Fabrycky, D.C., Ford, E.B., Fortney, J.J., Gilliland, R.L., Isaacson, H., Latham, D.W., Marcy, G.W., Quinn, S.N., Ragozzine, D., Shporer, A., Borucki, W.J., Ciardi, D.R., Gautier, T.N., III, Haas, M.R., Jenkins, J.M., Koch, D.G., Lissauer, J.J., Rapin, W., Basri, G.S., Boss, A.P., Buchhave, L.A., Carter, J.A., Charbonneau, D., Christensen-Dalsgaard, J., Clarke, B.D., Cochran, W.D., Demory, B.-O., Desert, J.-M., Devore, E., Doyle, L.R., Esquerdo, G.A., Everett, M., Fressin, F., Geary, J.C., Girouard, F.R., Gould, A., Hall, J.R., Holman, M.J., Howard, A.W., Howell, S.B., Ibrahim, K.A., Kinemuchi, K., Kjeldsen, H., Klaus, T.C., Li, J., Lucas, P.W., Meibom, S., Morris, R.L., Prša, A., Quintana, E., Sanderfer, D.T., Sasselov, D., Seader, S.E., Smith, J.C., Steffen, J.H., Still, M., Stumpe, M.C., Tarter, J.C., Tenenbaum, P., Torres, G., Twicken, J.D., Uddin, K., Van Cleve, J., Walkowicz, L., and Welsh, W.F. (2012) Planetary candidates observed by Kepler. III. Analysis of the first 16 months of data. Astrophys J Suppl Ser 204, doi:10.1088/0067-0049/204/2/24.

Beaulieu, J.P., Kipping, D.M., Batista, V., Tinetti, G., Ribas, I., Carey, S., Noriega-Crespo, J.A., Griffith, C.A., Campanella, G., Dong, S., Tennyson, J., Barber, R.J., Deroo, P., Fossey, S.J., Liang, D., Swain, M.R., Yung, Y., and Allard, N. (2010) Water in HD 209458b's atmosphere from 3.6-8 microns IRAC photometric observations in primary transit. Mon Not R Astron Soc 409:963-974.

Ben-Jaffel, L. (2007) Exoplanet HD 209458b: inated hydrogen atmosphere but no sign of evaporation. Astrophys J 671:L61-L64.

Ben-Jaffel, L. and Sona Hosseini, S. (2010) On the existence of energetic atoms in the upper atmosphere of exoplanet HD 209458b. Astrophys J 709:1284-1296.

Berta, Z.K., Charbonneau, D., Désert, J.-M., Miller-Ricci, K.E., McCullough, P.R., Burke, C.J., Fortney, J.J., Irwin, J., Nutzman, P., and Homeier, D. (2012) The flat transmission spectrum of the super-Earth GJ1214b from wide field camera 3 on the Hubble Space Telescope. Astrophys J 747, doi:10.1088/0004$637 \mathrm{X} / 747 / 1 / 35$.
Bodenheimer, P. (1997) The role of dust in star and planet formation: theory. In From Stardust to Planetesimals, ASP Conference Series Vol. 122, edited by Y.J. Pendleton and A.G.G.M. Tielens, Astronomical Society of the Pacific, San Francisco, pp $37-48$.

Borucki, W.J., Koch, D.G., Basri, G., Batalha, N., Boss, A., Brown, T.M., Caldwell, D., Christensen-Dalsgaard, J., Cochran, W.D., DeVore, E., Dunham, E.W., Dupree, A.K., Gautier, T.N., III, Geary, J.C., Gilliland, R., Gould, A., Howell, S.B., Jenkins, J.M., Kjeldsen, H., Latham, D.W., Lissauer, J.J., Marcy, G.W., Monet, D.G., Sasselov, D., Tarter, J., Charbonneau, D., Doyle, L., Ford, E.B., Fortney, J., Holman, M.J., Seager, S., Steffen, J.H., Welsh, W.F., Allen, C., Bryson, S.T., Buchhave, L., Chandrasekaran, H., Christiansen, J.L., Ciardi, D., Clarke, B.D., Dotson, J.L., Endl, M., Fischer, D., Fressin, F., Haas, M., Horch, E., Howard, A., Isaacson, H., Kolodziejczak, J., Li, J., MacQueen, P., Meibom, S., Prša, A., Quintana, E.V., Rowe, J., Sherry, W., Tenenbaum, P., Torres, G., Twicken, J.D., Van Cleve, J., Walkowicz, L., and Wu, H. (2011) Characteristics of Kepler planetary candidates based on the first data set. Astrophys J 728, doi:10.1088/0004-637X/728/2/117.

Borucki, W.J., Agol, E., Fressin, F., Kaltenegger, L., Rowe, J., Isaacson, H., Fischer, D., Batalha, N., Lissauer, J.J., Marcy, G.W., Fabrycky, D., Désert, J.M., Bryson, S.T., Barclay, T., Bastien, F., Boss, A., Brugamyer, E., Buchhave, L.A., Burke, C., Caldwell, D.A., Carter, J., Charbonneau, D., Crepp, J.R., Christensen-Dalsgaard, J., Christiansen, J.L., Ciardi, D., Cochran, W.D., DeVore, E., Doyle, L., Dupree, A.K., Endl, M., Everett, M.E., Ford, E.B., Fortney, J., Gautier, T.N., III, Geary, J.C., Gould, A., Haas, M., Henze, C., Howard, A.W., Howell, S.B., Huber, D., Jenkins, J.M., Kjeldsen, H., Kolbl, R., Kolodziejczak, J., Latham, D.W., Lee, B.L., Lopez, E., Mullally, F., Orosz, J.A., Prsa, A., Quintana, E.V., Sanchis-Ojeda, R., Sasselov, D., Seader, S., Shporer, A., Steffen, J.H., Still, M., Tenenbaum, P., Thompson, S.E., Torres, G., Twicken, J.D., Welsh, W.F., and Winn J.N. (2013) Kepler-62: A five-planet system with planets of 1.4 and 1.6 Earth radii in the habitable zone. Science 340:587-590.

Boss, A.P. (2003) Rapid formation of outer giant planets by disk instability. Astrophys J 599:577-581.

Charbonneau, D., Knutson, H.A., Barman, T., Allen, L.E., Mayor, M., Megeath, S.T., Queloz, D., and Udry, S. (2008) The broadband infrared emission spectrum of the exoplanet HD 189733b. Astrophys J 686:1341-1348.

Charbonneau, D., Berta, Z.K., Irwin, J., Burke, C.J., Nutzman, P., Buchhave, L.A., Lovis, C., Bonfils, X., Latham, D.W., Udry, S., Murray-Clay, R.A., Holman, M.J., Falco, E.E., Winn, J.N., Queloz, D., Pepe, F., Mayor, M., Delfose, X., and Forveille, T. (2009) A super-Earth transiting a nearby low-mass star. Nature 462:891-894.

Chassefière, E. (1996) Hydrodynamic escape of oxygen from primitive atmospheres: applications to the cases of Venus and Mars. Icarus 124:537-552.

Claire, M.W., Sheets, J., Cohen, M., Ribas, I., Meadows, V.S., and Catling, D.C. (2012) The evolution of solar flux from $0.1 \mathrm{~nm}$ to $160 \mu \mathrm{m}$ : quantitative estimates for planetary studies. Astrophys J 757, doi:10.1088/0004-637X/757/1/95.

Dorren, J.D. and Guinan, E.F. (1994) The Sun in time: detecting and modeling magnetic inhomogenities on solar-type stars. In The Sun as a Variable Star: Solar and Stellar Irradiance Variations: Proceedings of IAU Colloquium no. 143, Held in Boulder, Colorado, USA, June 20-25, 1993, edited by J.M. Pap, C. Fröhlich, H.S. Hudson, and S.K. Solanki, Cambridge University Press, New York, pp 206-216. 
Dorren, J.D., Guedel, M., and Guinan, E.F. (1995) X-ray emission from the Sun in its youth and old age. Astrophys J 448:431-436.

Dressing, C.D. and Charbonneau, D. (2013) The occurrence rate of small planets around small stars. Astrophys J, 767(1): article id. 95, 20 pp, arXiv:1302.1647D.

Dumusque, X., Pepe, F., Lovis, C., Ségransan, D., Sahlmann, J., Benz, W., Bouchy, F., Mayor, M., Queloz, D., Santos, N., and Udry, S. (2012) An Earth-mass planet orbiting $\alpha$ Centauri B. Nature 491:207-211.

Elkins-Tanton, L.T. (2008) Linked magma ocean solidification and atmospheric growth for Earth and Mars. Earth Planet Sci Lett 271:181-191.

Elkins-Tanton, L.T. (2011) Formation of water ocean on rocky planets. Astrophys Space Sci 332:359-364.

Elkins-Tanton, L. and Seager, S. (2008) Ranges of atmospheric mass and composition of super-Earth exoplanets. Astrophys $J$ 685:1237-1246.

Fossati, L., Haswell, C.A., Froning, C.S., Hebb, L., Holmes, S., Kolb, U., Helling, C., Carter, A., Wheatley, P., Cameron, A.C., Loeillet, B., Pollacco, D., Street, R., Stempels, H.C., Simpson, E., Udry, S., Joshi, Y.C., West, R.G., Skillen, I., and Wilson, D. (2010) Metals in the exosphere of the highly irradiated planet WASP-12b. Astrophys J 714:L222-L227.

France, K., Linsky, J.L., Tian, F., Froning, C.S., and Roberge, A. (2012) Time-resolved ultraviolet spectroscopy of the M-dwarf GJ 876 exoplanetary system. Astrophys J 750, doi:10.1088/ 2041-8205/750/2/L32.

France, K., Froning, C.S., Linsky, J.L., Roberge, A., Stocke, J.T., Tian, F., Bushinsky, R., Désert, J.-M., Mauas, P., Vieytes, M., and Walkowicz, L.M. (2013) The ultraviolet radiation environment around $\mathrm{M}$ dwarf exoplanet host stars. Astrophys $J$ 763, doi:10.1088/0004-637X/763/2/149.

French, M., Becker, A., Lorenzen, W., Nettelmann, N., Bethkenhagen, M., Wicht, J., and Redmer, R. (2012) Ab initio simulations for the material properties along Jupiter's adiabat. Astrophys J Suppl Ser 202, doi:10.1088/0067-0049/ $202 / 1 / 5$.

García Muñoz, A. (2007) Physical and chemical aeronomy of HD 209458b. Planet Space Sci 55:1426-1455.

Gaudi, B.S. (2012) Microlensing surveys for exoplanets. Annu Rev Astron Astrophys 50:411-453.

Gaudi, B.S. and Winn, J.N. (2007) Prospects for the characterization and confirmation of transiting exoplanets via the Rossiter-McLaughlin effect. Astrophys J 655:550-563.

Grießmeier, J.-M., Stadelmann, A., Penz, T., Lammer, H., Selsis, F., Ribas, I., Guinan, E.F., Motschmann, U., Biernat, H.K., and Weiss, W.W. (2004) The effect of tidal locking on the magnetospheric and atmospheric evolution of "Hot Jupiters." Astron Astrophys 425:753-762.

Grillmair, C.J., Burrows, A., Charbonneau, D., Armus, L., Stauffer, J., Meadows, V., van Cleve, J., von Braun, K., and Levine, D. (2008) Strong water absorption in the dayside emission spectrum of the planet HD189733b. Nature 456:767769 .

Güdel, M., Guinan, E.F., Mewe, R., Kaastra, J.S., and Skinner, S.L. (1997) A determination of the coronal emission measure distribution in the young solar analog EK Draconis from ASCA/EUVE spectra. Astrophys J 479:416-426.

Hamano, K., Abe, Y., and Genda, H. (2013) Emergence of two types of terrestrial planet on solidification of magma ocean. Nature 497:607-611.

Hayashi, C., Nakazawa, K., and Mizuno, H. (1979) Earth's melting due to the blanketing effect of the primordial dense atmosphere. Earth Planet Sci Lett 43:22-28.
Howard, A.W., Marcy, G.W., Bryson, S.T., Jenkins, J.M., Rowe, J.F., Batalha, N.M., Borucki, W.J., Koch, D.G., Dunham, E.W., Gautier, T.N., III, Van Cleve, J., Cochran, W.D., Latham, D.W., Lissauer, J.J., Torres, G., Brown, T.M., Gilliland, R.L., Buchhave, L.A., Caldwell, D.A., Christensen-Dalsgaard, J., Ciardi, D., Fressin, F., Haas, M.R., Howell, S.B., Kjeldsen, H., Seager, S., Rogers, L., Sasselov, D.D., Steffen, J.H., Basri, G.S., Charbonneau, D., Christiansen, J., Clarke, B., Dupree, A., Fabrycky, D.C., Fischer, D.A., Ford, E.B., Fortney, J.J., Tarter, J., Girouard, F.R., Holman, M.J., Johnson, J.A., Klaus, T.C., Machalek, P., Moorhead, A.V., Morehead, R.C., Ragozzine, D., Tenenbaum, P., Twicken, J.D., Quinn, S.N., Isaacson, H., Shporer, A., Lucas, P.W., Walkowicz, L.M., Welsh, W.F., Boss, A., Devore, E., Gould, A., Smith, J.C., Morris, R.L., Prša, A., Morton, T.D., Still, M., Thompson, S.E., Mullally, F., Endl, M., and MacQueen, P.J. (2012) Planet occurrence within 0.25 AU of solar-type stars from Kepler. Astrophys J Suppl Ser 201, doi:10.1088/0067-0049/201/2/15

Ikoma, M. and Genda, H. (2006) Constraints on the mass of a habitable planet with water of nebular origin. Astrophys $J$ 648:696-706.

Ikoma, M. and Hori, Y. (2012) In situ accretion of hydrogen-rich atmospheres on short-period super-Earths: implications for the Kepler-11 planets. Astrophys J 753, doi:10.1088/0004637X/753/1/66.

Ikoma, M., Nakazawa, K., and Emori, H. (2000) Formation of giant planets: dependences on core accretion rate and grain opacity. Astrophys J 537:1013-1025.

Jarosewich, E. (1990) Chemical analyses of meteorites: a compilation of stony and iron meteorite analyses. Meteoritics 25:323-337.

Kasting, J.F. (1995) $\mathrm{O}_{2}$ concentrations in dense primitive atmospheres: commentary. Planet Space Sci 43:11-13.

Kley, W. and Nelson, R.P. (2012) Planet-disk interaction and orbital evolution. Annu Rev Astron Astrophys 50:211-249.

Knutson, H.A., Charbonneau, D., Allen, L.E., Fortney, J.J., Agol, E., Cowan, N.B., Showman, A.P., Cooper, C.S., and Megeath, S.T. (2007) A map of the day-night contrast of the extrasolar planet HD 189733b. Nature 447:183-186.

Knutson, H.A., Charbonneau, D., Cowan, N.B., Fortney, J.J., Showman, A.P., Agol, E., Henry, G.W., Everett, M.E., and Allen, L.E. (2009) Multiwavelength constraints on the daynight circulation pattern of HD 189733b. Astrophys J 690:822836.

Kokubo, E. and Ida, S. (2000) Formation of protoplanets from planetesimals in the solar nebula. Icarus 143:15-27.

Koskinen, T.T., Harris, M.J., Yelle, R.V., and Lavvas, P. (2013a) The escape of heavy atoms from the ionosphere of HD209458b. I. A photochemical-dynamical model of the thermosphere. Icarus, in press, doi:10.1016/j.icarus.2012 .09.027.

Koskinen, T.T., Yelle, R.V., Harris, M.J., and Lavvas, P. (2013b) The escape of heavy atoms from the ionosphere of HD209458b. II. Interpretation of the observations. Icarus, in press, doi:10.1016/j.icarus.2012.09.026.

Kramm, U., Nettelmann, N., Fortney, J.J., Neuhäuser, R., and Redmer, R. (2012) Constraining the interior of extrasolar giant planets with the tidal Love number $\mathrm{k} 2$ using the example of HAT-P-13b. Astron Astrophys 538, doi:10.1051/0004-6361/ 201118141.

Kraus, A.L. and Ireland, M.J. (2012) LkCa 15: A young exoplanet caught at formation? Astrophys J 745, doi:10.1088/0004-637X/ 745/1/5.

Kuchner, M.J. (2003) Volatile-rich Earth-mass planets in the habitable zone. Astrophys J 596:L105-L108. 
Lammer, H. (2013) Origin and Evolution of Planetary Atmospheres: Implications for Habitability, Springer Briefs in Astronomy, Springer, Heidelberg.

Lammer, H., Bredehöft, J.H., Coustenis, A., Khodachenko, M.L., Kaltenegger, L., Grasset, O., Prieur, D., Raulin, F., Ehrenfreund, P., Yamauchi, M., Wahlund, J.-E., Grießmeier, J.-M., Stangl, G., Cockell, C.S., Kulikov, Yu.N., Grenfell, L., and Rauer, H. (2009) What makes a planet habitable? Astronomy and Astrophysics Review 17:181-249.

Lammer, H., Eybl, V., Kislyakova, K.G., Weingrill, J., Holmström, M., Khodchenko, M.L., Kulikov, Yu.N., Reiners, A., Leitzinger, M., Odert, P., Xian Grüß, M., Dorner, B., Güdel, M., and Hanslmeier, A. (2011) UV transit observations of EUV-heated expanded thermospheres of Earth-like exoplanets around M-stars: testing atmosphere evolution scenarios. Astrophys Space Sci 335:39-50.

Lammer, H., Kislyakova, K.G., Odert, P., Leitzinger, M., Schwarz, R., Pilat-Lohinger, E., Kulikov, Yu.N., Khodachenko, M.L., Güdel, M., and Hanslmeier, A. (2012) Pathways to Earth-like atmospheres: extreme ultraviolet (EUV)-powered escape of hydrogen-rich protoatmospheres. Orig Life Evol Biosph 41:503-522.

Lammer, H., Erkaev, N.V., Odert, P., Kislyakova, K.G., Leitzinger, M., and Khodachenko, M.L. (2013) Probing the blow-off criteria of hydrogen-rich "super-Earths." Mon Not R Astron Soc 430:1247-1256.

Lecavelier des Etangs, A., Ehrenreich, D., Vidal-Madjar, A., Ballester, G.E., Désert, J.-M., Ferlet, R., Hébrard, G., Sing, D.K., Tchakoumegni, K.-O., and Udry, S. (2010) Evaporation of the planet HD 189733b observed in H I Lyman- $\alpha$. Astron Astrophys 514:A72.

Lecavelier des Etangs, A., Bourrier, V., Wheatley, P.J., Dupuy, H., Ehrenreich, D., Vidal-Madjar, A., Hébrard, G., Ballester, G.E., Désert, J.-M., Ferlet, R., and Sing, D.K. (2012) Temporal variations in the evaporating atmosphere of the exoplanet HD 189733b. Astron Astrophys 543, doi:10.1051/0004-6361/ 201219363.

Léger, A., Rouan, D., Schneider, J., Barge, P., Fridlund, F., Samuel, B., Ollivier, M., Guenther, E., Deleuil, M., Deeg, H.J., Auvergne, M., Alonso, R., Aigrain, S., Alapini, A., Almenara, J.M., Baglin, A., Barbieri, M., Bruntt, H., Bordé, P., Bouchy, F., Cabrera, J., Catala, C., Carone, L., Carpano, S., Csizmadia, Sz., Dvorak, R., Erikson, A., Ferraz-Mello, S., Foing, B., Fressin, F., Gandolfi, D., Gillon, M., Gondoin, Ph., Grasset, O., Guillot, T., Hatzes, A., Hébrard, G., Jorda, L., Lammer, H., Llebaria, A., Loeillet, B., Mayor, M., Mazeh, T., Moutou, C., Pätzold, M., Pont, F., Queloz, D., Rauer, H., Renner, S., Samadi, R., Shporer, A., Sotin, Ch., Tingley, B., Wuchterl, G., Adda, M., Agogu, P., Appourchaux, T., Ballans, H., Baron, P., Beaufort, T., Bellenger, R., Berlin, R., Bernardi, P., Blouin, D., Baudin, F., Bodin, P., Boisnard, L., Boit, L., Bonneau, F., Borzeix, S., Briet, R., Buey, J.-T., Butler, B., Cailleau, D., Cautain, R., Chabaud, P.-Y., Chaintreuil, S., Chiavassa, F., Costes, V., Cuna Parrho, V., de Oliveira Fialho, F., Decaudin, M., Defise, J.-M., Djalal, S., Epstein, G., Exil, G.-E., Fauré, C., Fenouillet, T., Gaboriaud, A., Gallic, A., Gamet, P., Gavalda, P., Grolleau, E., Gruneisen, R., Gueguen, L., Guis, V., Guivarc'h, V., Guterman, P., Hallouard, D., Hasiba, J., Heuripeau, F., Huntzinger, G., Hustaix, H., Imad, C., Imbert, C., Johlander, B., Jouret, M., Journoud, P., Karioty, F., Kerjean, L., Lafaille, V., Lafond, L., Lam-Trong, T., Landiech, P., Lapeyrere, V., Larqué, T., Laudet, P., Lautier, N., Lecann, H., Lefevre, L., Leruyet, B., Levacher, P., Magnan, A., Mazy, E., Mertens, F., Mesnager, J.-M., Meunier, J.-C., Michel, J.-P., Monjoin, W., Naudet, D., Nguyen-Kim, K.,
Orcesi, J.-L., Ottacher, H., Perez, R., Peter, G., Plasson, P., Plesseria, J.-Y., Pontet, B., Pradines, A., Quentin, C., Reynaud, J.-L., Rolland, G., Rollenhagen, F., Romagnan, R., Russ, N., Schmidt, R., Schwartz, N., Sebbag, I., Sedes, G., Smit, H., Steller, M.B., Sunter, W., Surace, C., Tello, M., Tiphène, D., Toulouse, P., Ulmer, B., Vandermarcq, O., Vergnault, E., Vuillemin, A., and Zanatta, P. (2009) Transiting exoplanets from the CoRoT space mission VIII. CoRoT-7b: The first super-Earth with measured radius. Astron Astrophys 506:287302.

Lichtenegger, H.I.M., Lammer, H., Grießmeier, J.-M., Kulikov, Yu.N., von Paris, P., Hausleitner, W., Krauss, S., and Rauer, H. (2010) Aeronomical evidence for higher $\mathrm{CO}_{2}$ levels during Earth's Hadean epoch. Icarus 210:1-7.

Linsky, J.L., Yang, H., France, K., Froning, C.S., Green, J.C., Stocke, J.T., and Osterman, S.N. (2010) Observations of mass loss from the transiting exoplanet HD 209458b. Astrophys J 717:1291-1299.

Linsky, J.L., France, K., and Ayres, T. (2013) Computing intrinsic LY $\alpha$ fluxes of F5 V to M5 V stars. Astrophys J 766, doi:10.1088/ 0004-637X/766/2/69.

Lissauer, J.J., Fabrycky, D.C., Ford, E.B., Borucki, W.J., Fressin, F., Marcy, G.W., Orosz, J.A., Rowe, J.F., Torres, G., Welsh, W.F., Batalha, N.M., Bryson, S.T., Buchhave, L.A., Caldwell, D.A., Carter, J.A., Charbonneau, D., Christiansen, J.L., Cochran, W.D., Desert, J.-M., Dunham, E.W., Fanelli, M.N., Fortney, J.J., Gautier, T.N., III, Geary, J.C., Gilliland, R.L., Haas, M.R., Hall, J.R., Holman, M.J., Koch, D.G., Latham, D.W., Lopez, E., McCauliff, S., Miller, N., Morehead, R.C., Quintana, E.V., Ragozzine, D., Sasselov, D., Short, D.R., and Steffen, J.H. (2011) A closely packed system of low-mass, lowdensity planets transiting Kepler-11. Nature 470:53-58.

Lunine, J.I., O'Brien, D.P., Raymond, S.N., Morbidelli, A., Qinn, T., and Graps, A.L. (2011) Dynamical models of terrestrial planet formation. Adv Sci Lett 4:325-338.

Mannings, V., Boss, A.P., and Russell, S.S. (2000) Protostars and Planets IV, University of Arizona Press, Tucson.

Miller-Ricci, E. and Fortney, J.J. (2010) The nature of the atmosphere of the transiting super-Earth GJ 1214b. Astrophys J 716:L74-L79.

Mizuno, H. (1980) Formation of the giant planets. Progress of Theoretical Physics 64:544-557.

Montmerle, T., Augereau, J.-C., Chaussidon, M., Gounelle, M., Marty, B., and Morbidelli, A., (2006) 3. Solar System formation and early evolution: the first 100 million years. Earth Moon Planets 98:39-95.

Morbidelli, A., Lunine, J.I., O'Brien, D.P., Raymond, S.N., and Walsh, K.J. (2012) Building terrestrial planets. Annu Rev Earth Planet Sci 40:251-275.

Mumma, M.J. and Charnley, S.B. (2011) The chemical composition of comets-emerging taxonomies and natal heritage. Annu Rev Astron Astrophys 49:471-524.

Nettelmann, N. (2011) Predictions on the core mass of Jupiter and of giant planets in general. Astrophys Space Sci 336:47-51.

Nettelmann, N., Fortney, J.J., Kramm, U., and Redmer, R. (2011) Thermal evolution and structure models of the transiting super-Earth GJ 1214b. Astrophys J 733, doi:10.1088/0004-637X/ $733 / 1 / 2$.

Nettelmann, N., Becker, A., Holst, B., and Redmer, R. (2012) Jupiter models with improved ab initio hydrogen equation of state (H-REOS.2). Astrophys J 750, doi:10.1088/0004-637X/ $750 / 1 / 52$.

Penz, T., Erkaev, N.V., Kulikov, Yu.N., Langmayr, D., Lammer, H., Micela, G., Cecchi-Pestellini, C., Biernat, H.K., Selsis, F., 
Barge, P., Deleuil, M., and Léger, A. (2008) Mass loss from "Hot Jupiters": implications for CoRoT discoveries, part II: long time thermal atmospheric evaporation modeling. Planet Space Sci 56:1260-1272.

Rafikov, R.R. (2006) Atmospheres of protoplanetary cores: critical mass for nucleated instability. Astrophys J 648:666-682.

Raymond, S.N., Quinn, T., and Lunine, J.I. (2004) Making other Earths: dynamical simulations of terrestrial planet formation and water delivery. Icarus 168:1-17.

Raymond, S.N., O'Brien, D.P., Alessandro, M., and Kaib, N.A. (2009) Building the terrestrial planets: constrained accretion in the inner Solar System. Icarus 203:644-662.

Ribas, I., Guinan, E.F., Güdel, M., and Audard, M. (2005) Evolution of the solar activity over time and effects on planetary atmospheres. I. High-energy irradiances (1-1700 Å), Astrophys J 622:680-694.

Rogers, L.A. and Seager, S. (2010) Three possible origins for the gas layer on GJ 1214b. Astrophys J 716:1208-1216.

Sándor, Zs., Kley, W., and Klagyivik, P. (2007) Stability and formation of the resonant system HD 73526. Astron Astrophys 472:981-992.

Scalo, J., Kaltenegger, L., Segura, A.G., Fridlund, M., Ribas, I., Kulikov, Yu.N., Grenfell, J.L., Rauer, H., Odert, P., Leitzinger, M., Selsis, F., Khodachenko, M.L., Eiroa, C., Kasting, J., and Lammer, H. (2007) M stars as targets for terrestrial exoplanet searches and biosignature detection. Astrobiology 7:85-166.

Schneider, J., Rauer, H., Lasota, J.P., Bonazzola, S., and Chassefière, E. (1998) Brown dwarfs and extrasolar planets. In ASP Conf. Series 134, Astronomical Society of the Pacific, edited by R. Rebolo, E.L. Martin, and M.R. Zapatero Osorio, Provo, Utah, USA, pp 241-244.

Seager, S. and Deming, D. (2010) Exoplanet atmospheres. Annu Rev Astron Astrophys 48:631-672.

Seager, S., Deming, D., and Valenti, J.A. (2009) Transiting exoplanets with JWST. In Astrophysics in the Next Decade, Astrophysics and Space Science Proceedings, edited by H.A. Thomson, M. Stiavelli, and A.G.G.M. Tielens, Springer, Dordrecht, pp 123-145.

Selsis, F., Kasting, J.F., Levrard, B., Paillet, J., Ribas, I., and Delfosse, X. (2007) Habitable planets around the star Gliese 581? Astron Astrophys 476:1373-1387.

Shustov, B., Sachov, M., Gomez de Castro, A.I., Ana, I., and Pagano, I. (2009) WSO-UV ultraviolet mission for the next decade. Astrophys Space Sci 320:187-190.

Sozzetti, A. (2010) The Gaia Astrometric Survey. IAU Highlights of Astronomy 15:716-717.

Strassmeier, K.G. (2009) Starspots. Astronomy \& Astrophysics Review 17:251-308.

Swain, M.R., Tinetti, G., Vasisht, G., Deroo, P., Griffith, C., Bouwman, J., Chen, P., Yung, Y., Burrows, A., Brown, L.R., Matthews, J., Rowe, J.F., Kuschnig, R., and Angerhausen, D. (2009) Water, methane, and carbon dioxide present in the dayside spectrum of the exoplanet HD 209458b. Astrophys J 704:L1616-L1621.

Tian, F., Kasting, J.F., Liu, H., and Roble, R.G. (2008a) Hydrodynamic planetary thermosphere model: 1 . The response of the Earth's thermosphere to extreme solar EUV conditions and the significance of adiabatic cooling. J Geophys Res 113, doi:10.1029/2007JE002946.
Tian, F., Solomon, S.C., Qian, L., Lei, J., and Roble, R.G. (2008b) Hydrodynamic planetary thermosphere model: 2. Coupling of an electron transport/energy deposition model. J Geophys Res 113, doi:10.1029/2007JE003043.

Tinetti, G., Vidal-Madjar, A., Liang, M.C., Beaulieu, J.P., Yung, Y.L., Carey, S., Barber, R.J., Tennyson, J., Ribas, I., Allard, N., Ballester, G.E., Sing, D.K., and Selsis, F. (2007) Water vapour in the atmosphere of a transiting extrasolar planet. Nature 448:163-171.

Vidal-Madjar, A., Lecavelier des Etangs, A., Désert, J.M., Ballester, G.E., Ferlet, R., Hébrard, G., and Mayor, M., (2003) An extended upper atmosphere around the extrasolar planet HD209458b. Nature 422:143-146.

Vidal-Madjar, A., Désert, J.M., Lecavelier des Etangs, A., Hébrard, G., Ballester, G.E., Ehrenreich, D., Ferlet, R., McConnell, J.C., Mayor, M., and Parkinson, C.D. (2004) Detection of oxygen and carbon in the hydrodynamically escaping atmosphere of the extrasolar planet HD 209458b. Astrophys J 604:L69-L72.

von Bloh, W., Bounama, C., Cuntz, M., and Frank, S. (2007) The habitability of super-Earths in Gliese 581. Astron Astrophys 476:1365-1371.

Walsh, K.J., Morbidelli, A., Raymond, S.N., O'Brien, D.P., and Mandell, A.M. (2011) A low mass for Mars from Jupiter's early gas-driven migration. Nature 475:206-209.

Williams, J.P. and Cieza, L.A. (2011) Protoplanetary disks and their evolution. Annu Rev Astron Astrophys 49:67-117.

Wood, B.E., Müller, H.-R., Zank, G., and Linsky, J.L. (2002) Measured mass loss rates of solar-like stars as a function of age and activity. Astrophys J 574:412-425.

Wood, B.E., Müller, H.-R., Zank, G.P., Linsky, J.L., and Redfield, S. (2005) New mass-loss measurements from astrospheric Ly- $\alpha$ absorption. Astrophys J 628:L143-L146.

Wuchterl, G. (1993) The critical mass for protoplanets revisitedmassive envelopes through convection. Icarus 106:323-334.

Wuchterl, G., Guillot, T., and Lissauer, J.J. (2000) Giant planet formation. In Protostars and Planets IV, edited by V. Mannings, A.P. Boss, and S.S. Russell, University of Arizona Press, Tucson, pp 1081-1109.

Yelle, R.V. (2004) Aeronomy of extra-solar giant planets at small orbital distances. Icarus 170:167-179.

Zaqarashvili, T.V., Oliver, R., Ballester, J.L., Carbonell, M., Khodachenko, M.L., Lammer, H., Leitzinger, M., and Odert, P. (2011) Rossby waves and polar spots in rapidly rotating stars: implications for stellar wind evolution. Astron Astrophys 532:A139.

Address correspondence to: Helmut Lammer

Austrian Academy of Sciences Space Research Institute Schmiedlstr. 6 A-8042 Graz Austria

E-mail: helmut.lammer@oeaw.ac.at

Submitted 9 March 2013 Accepted 22 June 2013 